\title{
The tertiary structure of the human Xkr8-Basigin complex that scrambles phospholipids at plasma
} membranes

\author{
Takaharu Sakuragi ${ }^{1}$, Ryuta Kanai' ${ }^{2}$ Akihisa Tsutsumi ${ }^{3}$, Hirotaka Narita ${ }^{4,7}$, Eriko Onishi1, \\ Kohei Nishino5, Takuya Miyazaki ${ }^{6}$, Takeshi Baba6, Hidetaka Kosako5, Atsushi Nakagawa ${ }^{4}{ }^{4}$, \\ Masahide Kikkawa $\mathbb{1}^{3}$, Chikashi Toyoshima $\mathbb{B}^{2}$ and Shigekazu Nagata $\mathbb{1}^{1 凶}$
}

\begin{abstract}
Xkr8-Basigin is a plasma membrane phospholipid scramblase activated by kinases or caspases. We combined cryo-EM and $X$-ray crystallography to investigate its structure at an overall resolution of $3.8 \AA$. Its membrane-spanning region carrying 22 charged amino acids adopts a cuboid-like structure stabilized by salt bridges between hydrophilic residues in transmembrane helices. Phosphatidylcholine binding was observed in a hydrophobic cleft on the surface exposed to the outer leaflet of the plasma membrane. Six charged residues placed from top to bottom inside the molecule were essential for scrambling phospholipids in inward and outward directions, apparently providing a pathway for their translocation. A tryptophan residue was present between the head group of phosphatidylcholine and the extracellular end of the path. Its mutation to alanine made the Xkr8-Basigin complex constitutively active, indicating that it plays a vital role in regulating its scramblase activity. The structure of Xkr8-Basigin provides insights into the molecular mechanisms underlying phospholipid scrambling.
\end{abstract}

T he lipid bilayer in eukaryote plasma membranes comprises asymmetrically distributed phospholipids ${ }^{1}$. Phosphatidylserine (PtdSer) and phosphatidylethanolamine (PtdEtn) localize to the inner leaflet of the plasma membrane, and phosphatidylcholine (PtdCho) to the outer leaflet. This asymmetrical distribution is disrupted in various biological processes, and PtdSer exposed to the cell surface activates enzymes or functions as a signal ${ }^{2-4}$. Activated platelets expose PtdSer as a scaffold for clotting enzymes, while PtdSer exposed on apoptotic cells functions as an 'eat me' signal for macrophages.

ATP-dependent flippases (P4-ATPases) translocate PtdSer and PtdEtn from the outer to inner leaflet ${ }^{5}$ and maintain their asymmetrical distribution. A high concentration of $\mathrm{Ca}^{2+}$ in activated platelets or caspase in apoptotic cells inactivates flippases $^{5,6}$; however, this inactivation alone is insufficient for the swift exposure of PtdSer because it takes days for phospholipids carrying a hydrophilic head group to travel the hydrophobic lipid bilayer?. To expedite the exposure of PtdSer, cells carry scramblases that translocate phospholipids bidirectionally in an energy-independent manner ${ }^{2,5}$. We previously identified two membrane proteins (TMEM16F and Xkr8) as scramblases ${ }^{8,9}$ that are activated by distinct mechanisms. TMEM16F and several other TMEM16 family members, including their fugal homologs, function as $\mathrm{Ca}^{2+}$-dependent scramblases ${ }^{8,10-14}$. Xkr8 is a member of the XK family ${ }^{9}$ and forms a heterodimer with Basigin (BSG) or Neuroplastin $(\mathrm{NPTN})^{15}$. It is activated by kinase or caspase to scramble phospholipids ${ }^{9,16}$. Xkr8 is responsible for exposing PtdSer in apoptotic cells, and its deficiency causes systemic lupus erythematosus-type autoimmune disease and male infertility ${ }^{17,18}$.
The tertiary structures of P4-ATPases and TMEM16F family members were previously elucidated ${ }^{11,12,19-22}$, and various models, such as the 'stepping stone model', 'credit card model', 'out-of-the-groove model' and 'membrane distortion model', have been proposed ${ }^{23-27}$. We herein report the structure of the human Xkr8-BSG heterodimer with a novel fold. It carries 22 hydrophilic amino acids in the transmembrane region of the molecule. A set of hydrophilic residues was necessary for the stability of the complex, while the other six charged residues were essential for its scrambling activity. The molecule had a hydrophobic cleft occupied by PtdCho on an upper surface. The head group of PtdCho coordinated with a tryptophan residue at the extracellular end of a transmembrane helix. Reductions in the hydrophobicity of the cleft made the molecule inactive, while the replacement of tryptophan by alanine made the molecule constitutively active. These results provide insights into the phospholipid scrambling mechanism.

\section{Results}

Purification of the hXkr8-hBSG - -Fab complex. The amino acid sequence of Xkr8 is well conserved in vertebrates (Supplementary Fig. 1). Xkr8 requires BSG or NPTN to localize to the plasma membrane ${ }^{15}$. We initially screened GFP-tagged Xkr8 orthologues for their expression and stability using fluorescence-detection size-exclusion chromatography and found that human Xkr8 (hXkr8) and hBSG were relatively stable and abundantly expressed. BSG carries two immunoglobulin domains, one of which is dispensable for its chaperone-like activity ${ }^{15}$. We removed the first immunoglobulin and modified $N$-glycosylation sites (hBSG $\Delta$ ) (Extended Data Fig. 1a). hBSG $\Delta$ supported the translocation of hXkr8 to the plasma

\footnotetext{
'Laboratory of Biochemistry and Immunology, World Premier International Immunology Frontier Research Center, Osaka University, Suita, Japan. ${ }^{2}$ Institute for Quantitative Biosciences, The University of Tokyo, Tokyo, Japan. ${ }^{3}$ Department of Cell Biology and Anatomy, Graduate School of Medicine, The University of Tokyo, Tokyo, Japan. ${ }^{4}$ Institute for Protein Research, Osaka University, Suita, Japan. ${ }^{5}$ Division of Cell Signaling, Fujii Memorial Institute of Medical Sciences, Tokushima University, Tokushima, Japan. ${ }^{6}$ Research Division, Chugai Pharmaceutical Co., Ltd., Kamakura, Japan. ${ }^{7}$ Present address: Japan Aerospace Exploration Agency (JAXA), Tsukuba, Japan. 『e-mail: snagata@ifrec.osaka-u.ac.jp
} 

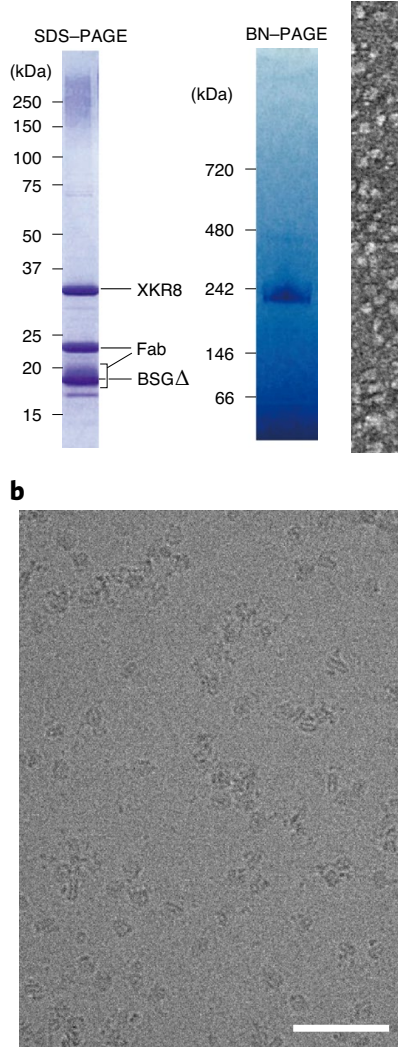

d

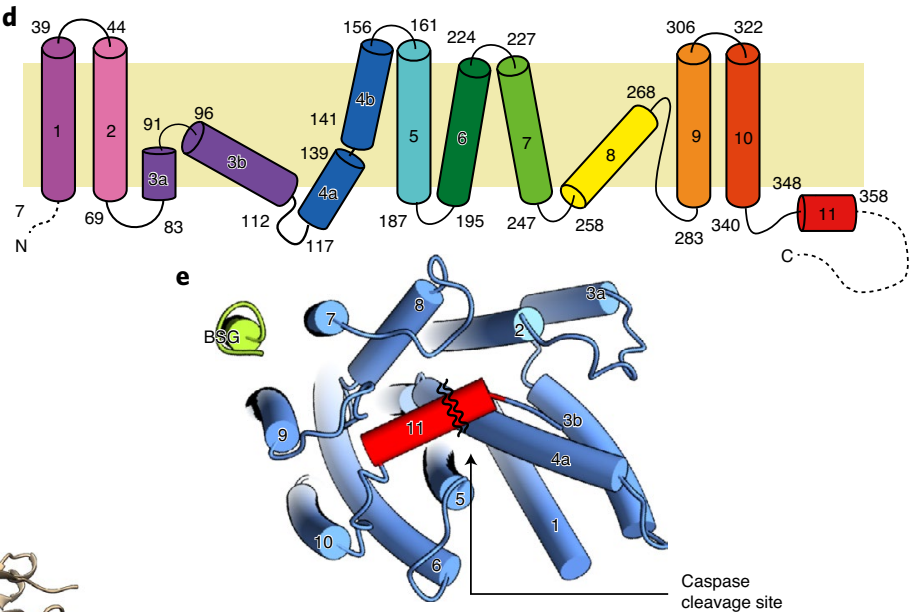

c

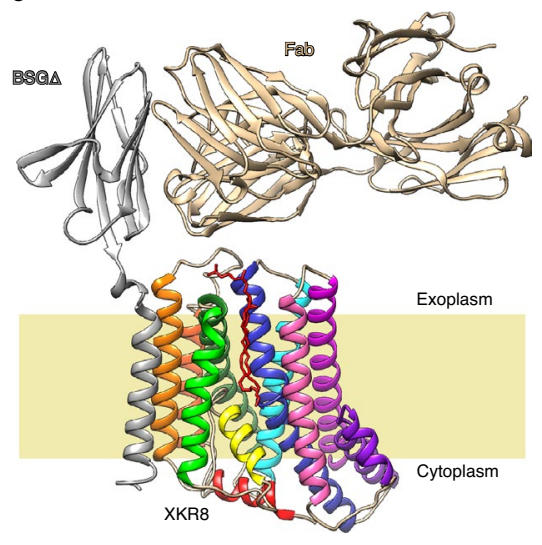

$\mathbf{f}$

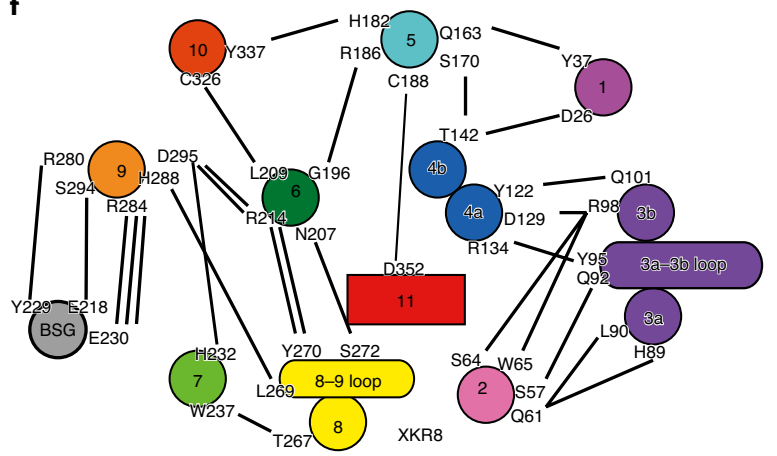

Fig. 1 | Structure of the hXkr8-hBSG $\Delta$ complex. a, Purification of the hXkr8-hBSG $\Delta$-Fab18 complex. The purified hXkr8-hBSG $\Delta$-Fab18 complex was analyzed by SDS-PAGE ( $3 \mu \mathrm{g})$ or blue native (BN)-PAGE $(5 \mu \mathrm{g})$, or observed under an electron microscope (HITACHI H7650) after negative staining. Bar, 100 nm.

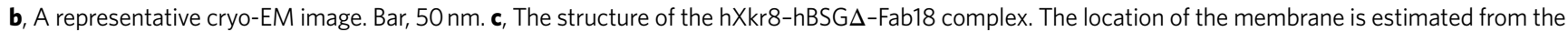
position of tryptophan (Extended Data Fig. 6). d, $\alpha$-Helices of $h X k r 8$ are numbered and schematically shown. $\mathbf{e}, A$ view of the $h X k r 8-h B S G \Delta$ complex from its cytoplasmic side. f, Amino acids connected via hydrogen bonds or salt bridges in the hXkr8-hBSG $\Delta$ complex are shown. Source data for $\mathbf{a}$ are available online.

membrane in $\mathrm{NPTN}^{-/-} \mathrm{BSG}^{-/-} \mathrm{W} 3(\mathrm{DKO})^{15}$ cells (Extended Data Fig. $1 \mathrm{~b})$, and transformed cells retained the ability to scramble phospholipids during apoptosis (Extended Data Fig. 1c).

hXkr8 was then fused to a histidine tag and EGFP and co-expressed with hBSG $\Delta$ in Sf9 cells using a baculovirus system. The hXkr8-hBSG $\Delta$ complex purified from the lauryl-maltose neopentyl glycol (LMNG)-solubilized membrane fraction was nearly homogeneous (Extended Data Fig. 1d). Since the Fab of a monoclonal antibody often enhanced the crystal formation of membrane proteins ${ }^{28}$, we prepared a Fab that preferentially recognized native over denatured hBSG $\Delta$. We initially attempted to crystallize the hXkr8-hBSG $\Delta$-Fab14 complex; however, it was not possible to visualize the electron density of the transmembrane regions in the crystals because these regions appeared to have been lost during crystallization. Nevertheless, X-ray crystallography of Fab14 and hBSG $\Delta$-Fab14 revealed the structure of the extracellular region of the hBSG $\Delta$-Fab14 complex at a high resolution of $2.51 \AA$ (Table 1 , Extended Data Fig. 2 and Supplementary Fig. 2). To elucidate the structures of the transmembrane regions of hXkr8 and hBSG, free LMNG was removed by GraDeR ${ }^{29}$ (Extended Data Fig. 1e), and nearly homogeneous monodispersed samples (Fig. 1a) were subjected to a cryo-EM single-particle analysis.

Structure of the hXkr8-hBSG complex. Despite the relatively small size of the hXkr8-hBSG $\Delta$-Fab18 complex $(110 \mathrm{kDa})$, electron micrographs revealed the structure of the hXkr8-hBSG $\Delta-$ Fab18 complex at a resolution of $3.8 \AA$, measured using a Fourier shell cor- relation (FSC) cut-off of 0.143 (Fig. 1b, Table 2 and Extended Data Fig. 3). Based on the density map obtained by cryo-EM (Extended Data Fig. 4) and the X-ray structure of hBSG $\Delta-$ Fab (Extended Data Fig. 5), we elucidated the structure of the hXkr8-hBSG $\Delta-$ Fab complex. It consisted of hXkr8, hBSG $\Delta$ and Fab at a 1:1:1 ratio (Fig. 1c). Based on the position of tryptophan residues near the end of the helices ${ }^{30,31}$ and surface hydrophobicity (Extended Data Fig. 6), we determined the location of the membrane. hXkr8 comprised eight transmembrane helices ( $\alpha 1, \alpha 2, \alpha 4-7, \alpha 9$ and $\alpha 10)$, two helices traveling halfway to the membrane ( $\alpha 3$ and $\alpha 8)$ and one cytoplasmic helix $(\alpha 11)$ (Fig. 1d). $\alpha 11$ contained a caspase 3-recognition sequence; interacted with $\alpha 2, \alpha 4, \alpha 5$ and $\alpha 6$ (Fig. 1e); and appeared to stabilize the disposition of these helices at the cytoplasmic side. The transmembrane region of hXkr8-hBSG $\Delta$ had a rectangular cuboid-like structure with a slight spread to the cytoplasmic face (Fig. 1c). Proteins with similar structures were not detected in a three-dimensional (3D) homology search (PDBeFold) ${ }^{32}$.

Interaction between hXkr8 and BSG. The hXkr8-hBSG structure and an analysis of hydrogen bonds and salt bridges indicated that $\alpha 9$ of hXkr8 was arranged near the transmembrane region of hBSG $\Delta$ (Fig. 1e,f). A207 and P211 of hBSG $\Delta$ were arranged toward T305 and T302 of hXkr8 at a distance of approximately 0.7 and $0.4 \mathrm{~nm}$, respectively (Fig. 2a). These residues were individually mutated to cysteine, tagged with GFP (for hXkr8) or hemagglutinin (HA) (for BSG) (Fig. 2b) and expressed in HEK293T cells. Western blotting under nonreducing conditions showed a $100-\mathrm{kDa}$ band 
Table 1 | Data collection and refinement statistics

\begin{tabular}{|c|c|c|}
\hline & Fab14 (PDB 7D9Z) & $\begin{array}{l}\text { hBSGext/Fab14 (PDB } \\
\text { 7DAA) }\end{array}$ \\
\hline \multicolumn{3}{|l|}{ Data collection } \\
\hline Space group & $C 2$ & $\mathrm{C} 222_{1}$ \\
\hline \multicolumn{3}{|l|}{ Cell dimensions } \\
\hline$a, b, c(\AA)$ & $163.45,53.05,54.31$ & $117.35,247.35,522.25$ \\
\hline$\alpha, \beta, \gamma\left(^{\circ}\right)$ & $90,105.05,90$ & $90,90,90$ \\
\hline Resolution $(\AA)$ & $\begin{array}{l}78.924-1.123 \\
(1.194-1.123)^{a}\end{array}$ & $\begin{array}{l}123.676-2.509 \\
(2.706-2.509)\end{array}$ \\
\hline$R_{\text {merge }}\left(\right.$ all $\mathrm{I}^{+}$and $\left.\mathrm{I}^{-}\right)$ & $0.048(0.653)$ & $0.187(1.569)$ \\
\hline$R_{\text {merge }}\left(\right.$ within $\left.\mathrm{I}^{+} / \mathrm{I}^{-}\right)$ & $0.045(0.659)$ & $0.178(1.421)$ \\
\hline$|/ \sigma|$ & $13.5(1.4)$ & $7.3(1.3)$ \\
\hline $\begin{array}{l}\text { Completeness } \\
\text { (spherical) (\%) }\end{array}$ & $81.6(24.3)$ & $68.4(10.8)$ \\
\hline $\begin{array}{l}\text { Completeness } \\
\text { (ellipsoidal) (\%) }\end{array}$ & $87.0(33.7)$ & $86.3(45.7)$ \\
\hline Redundancy & $4.8(3.1)$ & $5.1(7.2)$ \\
\hline \multicolumn{3}{|l|}{ Refinement } \\
\hline Resolution $(\AA)$ & $36.40-1.12(1.13-1.12)$ & $123.68-2.51(2.64-2.51)$ \\
\hline No. reflections & $139,266(526)$ & $18,231(204)$ \\
\hline$R_{\text {work }} / R_{\text {free }}$ & $\begin{array}{l}0.121(0.256) / 0.150 \\
(0.333)\end{array}$ & $\begin{array}{l}0.228(0.344) / 0.283 \\
(0.551)\end{array}$ \\
\hline \multicolumn{3}{|l|}{ No. atoms } \\
\hline Protein & 3,418 & 3,847 \\
\hline Ligand/ion & $37 / 0$ & $0 / 11$ \\
\hline Water & 695 & 0 \\
\hline \multicolumn{3}{|l|}{$B$ factors } \\
\hline Protein & 19.78 & 38.92 \\
\hline Ligand/ion & $21.50 /-$ & $-/ 118.45$ \\
\hline Water & 33.76 & - \\
\hline \multicolumn{3}{|l|}{ R.m.s. deviations } \\
\hline Bond lengths $(\AA)$ & 0.010 & 0.003 \\
\hline Bond angles $\left({ }^{\circ}\right)$ & 1.178 & 0.565 \\
\hline
\end{tabular}

The number of crystals for each structure is one. R.m.s., root mean square. ${ }^{a}$ Values in parentheses are for the highest-resolution shell.

with anti-GFP and anti-HA when hXkr8-T305C was co-expressed with hBSG-A207C (Fig. 2c). When the membrane fraction was treated with the oxidant before SDS-polyacrylamide gel electrophoresis (SDS-PAGE), P211C of hBSG $\Delta$ and T302C of hXkr8 were also crosslinked (Fig. 2c), confirming the proximity of these residues. At the cytoplasmic side of the hXkr8-hBSG $\Delta$ complex, E230 of hBSG $\Delta$ was close to Q247, R280 and R284 of hXkr8 (Fig. $2 \mathrm{~d})$. The E230A mutant of hBSG did not support the localization of hXkr8-GFP to the plasma membrane (Fig. 2e). The R284E mutant of hXkr8 did not localize to the plasma membrane in PLB cells expressing intact hBSG (Fig. 2e), indicating that the interaction between E230 of BSG and R284 of hXkr8 was indispensable for the chaperone activity of BSG.

A hydrophilic pathway inside the molecule essential for scrambling phospholipids. The amino acid sequence of mouse $(\mathrm{m}) \mathrm{Xkr} 8$ has $68.9 \%$ identity with hXkr8 (272 of 395 amino acids) (Fig. 3a). Using the Modeller program ${ }^{33}$, the homology model of the mXkr8 structure was predicted, and the two structures were superimposed
Table 2 | Cryo-EM data collection, refinement and validation statistics

hXkr8-hBSG $\Delta$-Fab18 complex

(EMD-30636) (PDB 7DCE)

\begin{tabular}{|c|c|}
\hline \multicolumn{2}{|l|}{ Data collection and processing } \\
\hline Magnification & 105,000 \\
\hline Voltage (kV) & 300 \\
\hline Electron exposure $\left(\mathrm{e}^{-} / \AA^{2}\right)$ & 48 \\
\hline Defocus range $(\mu \mathrm{m})$ & $0.8 \sim 1.8$ \\
\hline Pixel size $(\AA)$ & 0.83 \\
\hline Symmetry imposed & $\mathrm{Cl}$ \\
\hline Initial particle images (no.) & 864,702 \\
\hline Final particle images (no.) & 62,124 \\
\hline Map resolution $(\AA)$ & 3.8 \\
\hline FSC threshold & 0.143 \\
\hline Map resolution range $(\AA)$ & $3.67-6.51$ \\
\hline \multicolumn{2}{|l|}{ Refinement } \\
\hline Initial model used & De novo structure \\
\hline Map sharpening $B$ factor $\left(\AA^{2}\right)$ & -120 \\
\hline \multicolumn{2}{|l|}{ Model composition } \\
\hline Nonhydrogen atoms & 7,102 \\
\hline Protein residues & 912 \\
\hline Ligands & $1(\mathrm{DLP})$ \\
\hline \multicolumn{2}{|l|}{$B$ factors $\left(\AA^{2}\right)$} \\
\hline Protein & 84.16 \\
\hline Ligand & 66.09 \\
\hline \multicolumn{2}{|l|}{ R.m.s. deviations } \\
\hline Bond lengths $(\AA)$ & 0.009 \\
\hline Bond angles $\left({ }^{\circ}\right)$ & 1.203 \\
\hline \multicolumn{2}{|l|}{ Validation } \\
\hline MolProbity score & 2.45 \\
\hline Clashscore & 11.63 \\
\hline Poor rotamers (\%) & 3.27 \\
\hline \multicolumn{2}{|l|}{ Ramachandran plot } \\
\hline Favored (\%) & 92.34 \\
\hline Allowed (\%) & 7.44 \\
\hline Disallowed (\%) & 0.22 \\
\hline
\end{tabular}

with a root mean square deviation of $0.215 \AA$, suggesting that the structure of mXkr8 was essentially identical to hXkr8 (Extended Data Fig. 7). An interesting feature of hXkr8 was the presence of 22 charged residues (Asp, Glu, Lys and Arg) in the $\alpha$-helices at the lipid layer (Fig. 3a). Sixteen of these residues were well conserved among vertebrates (Supplementary Fig. 1). To examine the role of these charged residues, we selected 12 residues of $\mathrm{mXkr8}$ or hXkr8 (D12, D26, D30, R98, D129, K134, E137, E141, D180, D295, R183 and R214) that were internally oriented (Protein Data Bank (PDB) 7DCE). R214 and D295 were mutated to glycine or lysine, respectively, similar to XK in human patients with McLeod Syndrome ${ }^{34,35}$. The other ten residues were mutated to Ala. The mutants were tagged by GFP at the C terminus and stably expressed in PLB985 or $\mathrm{Ba} / \mathrm{F} 3$ cells. As shown in Fig. 3b, four mutants (R98A, D129A, $\mathrm{R} 214 \mathrm{G}$ and $\mathrm{D} 295 \mathrm{~K}$ ) were not localized at the plasma membrane. A western blot analysis showed that the expression of these mutant 
a

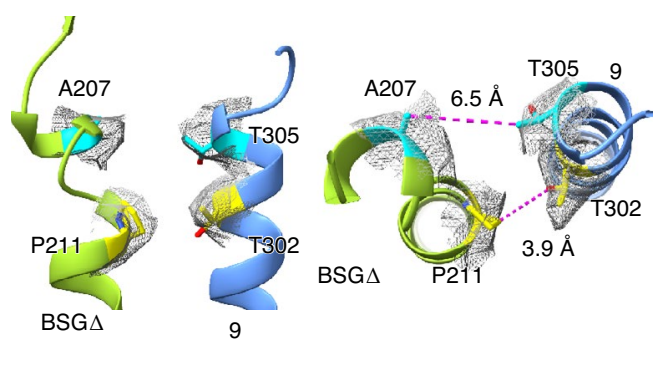

b

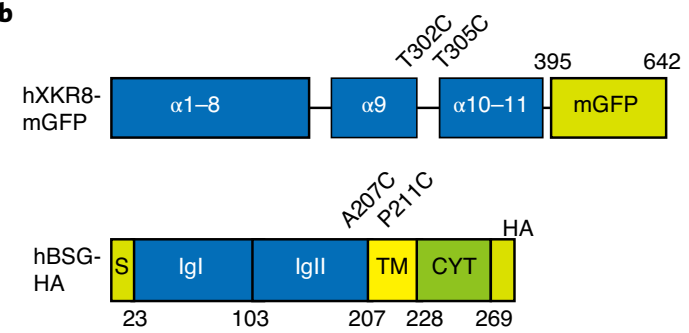

d

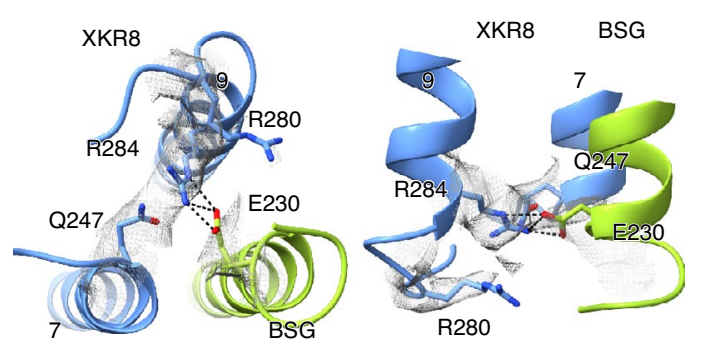

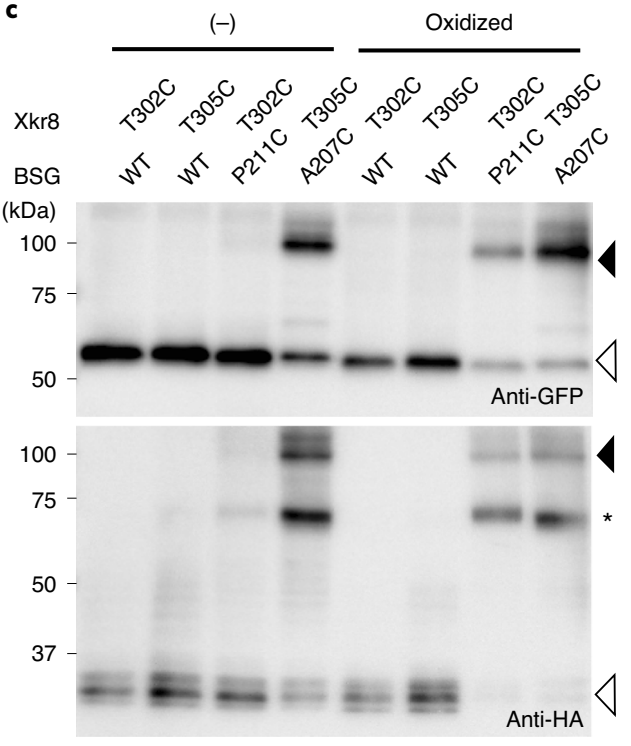
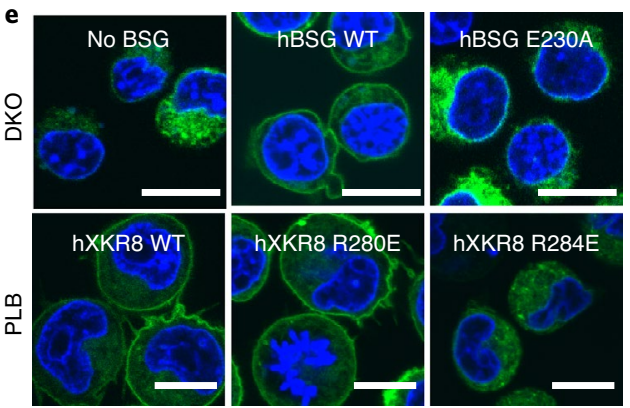

Fig. 2 | Interaction between hXkr8 and hBSG $\Delta$. a, Side (left) and top (right) views of hXkr8-hBSG $\Delta$ near the extracellular surface with local density (gray mesh) from the cryo-EM map. b, Crosslinking of cysteine-substituted hXkr8 and hBSG. The residues mutated to cysteine (T302 and T305 in hXkr8, and A207 and P211 in hBSG) are indicated in their schematic structure. c, Wild-type (WT) and indicated mutant hXkr8-mGFP and hBSG-HA were expressed in HEK293T cells. Crude membrane fractions were treated or not with copper phenanthroline for oxidization, and subjected to western blotting for GFP (upper panel) or HA (lower panel). Black arrowheads, crosslinked protein; white arrowheads, monomer; ${ }^{\star}$, BSG dimer ${ }^{47}$. d, The cytoplasmic (left) and side (right) views of the interaction between $\mathrm{hXkr8}$ and hBSG $\Delta$ near the cytoplasm, with local density from the cryo-EM map shown as gray mesh. R284 of Xkr8 is connected with E230 of hBSG via hydrogen bonds. e, DKO cells expressing hXkr8-GFP with the WT or E230A hBSG, or PLB cells expressing EGFP-tagged WT, R280E or R284E hXkr8, were observed for EGFP and Hoechst 33342. Scale bar, $10 \mu \mathrm{m}$. Source data for c and e are available online.

proteins was markedly reduced compared with the wild-type Xkr8 (Fig. 3c). The structure of hXkr8 indicated two clusters of hydrophilic amino acids (one group with R214, H232, Y270 and D295; and another with S64, R98 and D129) in the transmembrane region of the complex (Fig. 3d). R214 on $\alpha 6$ and D295 on $\alpha 9$ formed a salt bridge, while D129 in $\alpha 4$ a formed a salt bridge with R98 on $\alpha 3 \mathrm{~b}$. The reduced expression of the mutant proteins (R98A, D129A, D295K and $\mathrm{R} 214 \mathrm{G}$ ) indicated that the interaction between the hydrophilic residues in the transmembrane region plays an essential role in stabilizing the Xkr8-BSG complex.

The eight other mutants (D12A, D26A, D30A, K134A, E137A, E141A, D180A and R183A) were expressed as efficiently as wild-type mXkr8 and localized at plasma membranes (Fig. 3b,c). mXkr8-mediated lipid scrambling was activated in mouse $\mathrm{Ba} / \mathrm{F} 3$ cells by phosphorylation at the C-terminal tail region and PtdSer exposure occurred at $4{ }^{\circ} \mathrm{C}$, a temperature at which P4-ATPase activity should be strongly reduced ${ }^{16}$. We used this system to examine the scrambling activities of mXkr8 mutants. As shown in Fig. 4a, $\mathrm{Ba} / \mathrm{F} 3$ cell transformants expressing wild-type or D12A or D180A mutant mXkr8 exposed PtdSer. On the other hand, the exposure of PtdSer was markedly reduced in transformants expressing the D26A, D30A, K134A, E137A, E141A and R183A mutants, indicating that these residues play an indispensable role in scrambling
PtdSer from the inner to outer leaflets of plasma membranes. Xkr8 nonspecifically scrambles various phospholipids ${ }^{9}$. Accordingly, the treatment with cinnamycin (Ro 09-0918), which kills cells exposing $\mathrm{PtdEtn}^{36}$, lysed $\mathrm{Ba} / \mathrm{F} 3$ transformants expressing wild-type mXkr8, D12A, D180A and R183A at $4^{\circ} \mathrm{C}$ for $15 \mathrm{~min}$ (Fig. 4b,d). However, its killing activity was reduced in cells expressing the D26A, D30A, K134A, E137A or E141A mutants. Xkr8 scrambles phospholipids from the inner to outer leaflets and from the outer to inner leaflets?. Accordingly, wild-type mXkr8 and the D12A and D180A mutants efficiently internalized NBD-SM ((7S)-4-hydroxy-7-[(1R,2E)1-hydroxy-2-hexadecen-1-yl]-N,N,N-trimethyl-14-[(7-nitro2,1,3-benzoxadiazol-4-yl)amino]-9-oxo-3,5-dioxa-8-aza-4-phosph atetradecan-1-aminium, 4-oxide inner salt) at $4{ }^{\circ} \mathrm{C}$ (Extended Data Fig. 8). In contrast, the mutations in D26, D30, K134, E137, E141 and R183 markedly reduced this activity. Since sphingomyelin does not activate flippases (ATP11A and ATP11C) at plasma membranes $^{37}$, we assayed the incorporation of NBD-SM at $20^{\circ} \mathrm{C}$ (Fig. $4 \mathrm{c}, \mathrm{d}$ ). Consistent with the results obtained at $4^{\circ} \mathrm{C}, \mathrm{Ba} / \mathrm{F} 3$ cell transformants expressing wild-type Xkr8 efficiently incorporated NBD-SM. However, the six mutants exhibited negligible activity to internalize NBD-SM.

The structure of hXkr8 indicated that these six amino acids (D26, D30, R134, E137, E141 and R183) were arranged from top to 
a

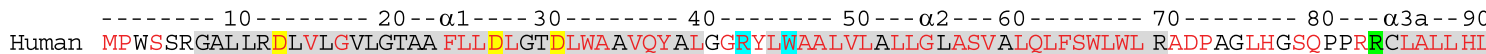
Mouse Mouse MPLSVHHHVALDVVVGLVSILS FLLDLVADLWAVVQYVLLGRYLWAALVLVLLGQASVLLQLFSWLWL TADP TELHHSQLSRPFLALL HI

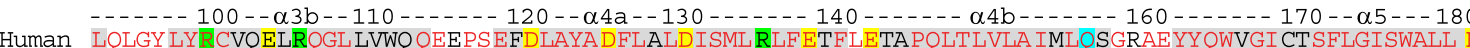

Mouse Mouse LQLGY LYRCLHGMHQGL SMCY QEMPSECDLAYADFLSLDISML KLFESFLEATPQLTLVLA IVLQNGQAEYYQWFGI SS SFLGISWALL D

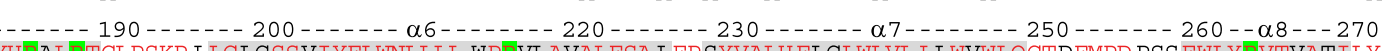
Human YHRALRTCLPSKP LLGLGSSVIYFLWNLLLL WPRVLAVALFSA LFP SYVALH FLGLWLVL LLWVWLQGTDFMPD PSS EWLYRVTVATILY Mouse YHRSLRTCLPSKP RLGRS SSAIYFLWNLLLL GPR ICAIALFSA VFPYYVALH FFSLWLVLLFWIWLQ GTNFMPD SKG EWLY RVTMALILY _.

Human FSWFNVAEGRTR GRAI IHFAFLLSDS ILLVA-TWVTHSSWLPSGI PLQLWLPVGCGCFFLGLAL RLVYYHWLHPSC CWKPDPDQVDGARS

Mouse FSWFNV SGGRTR GRAVIHLI F IF SDSVLLVTTSWVTH GTWLPSGI SLLMWVT IGGACFFLGLAL RVI YY LWLHPSC SW--DPDLVD GTLG

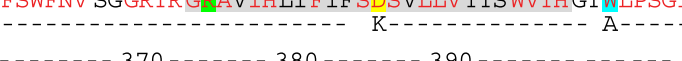

Human LLSPEGYQLPQNR RMTHLAQKF FPKAKDEAASPVKG -

Mouse LLSPHRPPKLIYN RRATLLAEN FF-AKAKARAVLTEEVQLNGVI
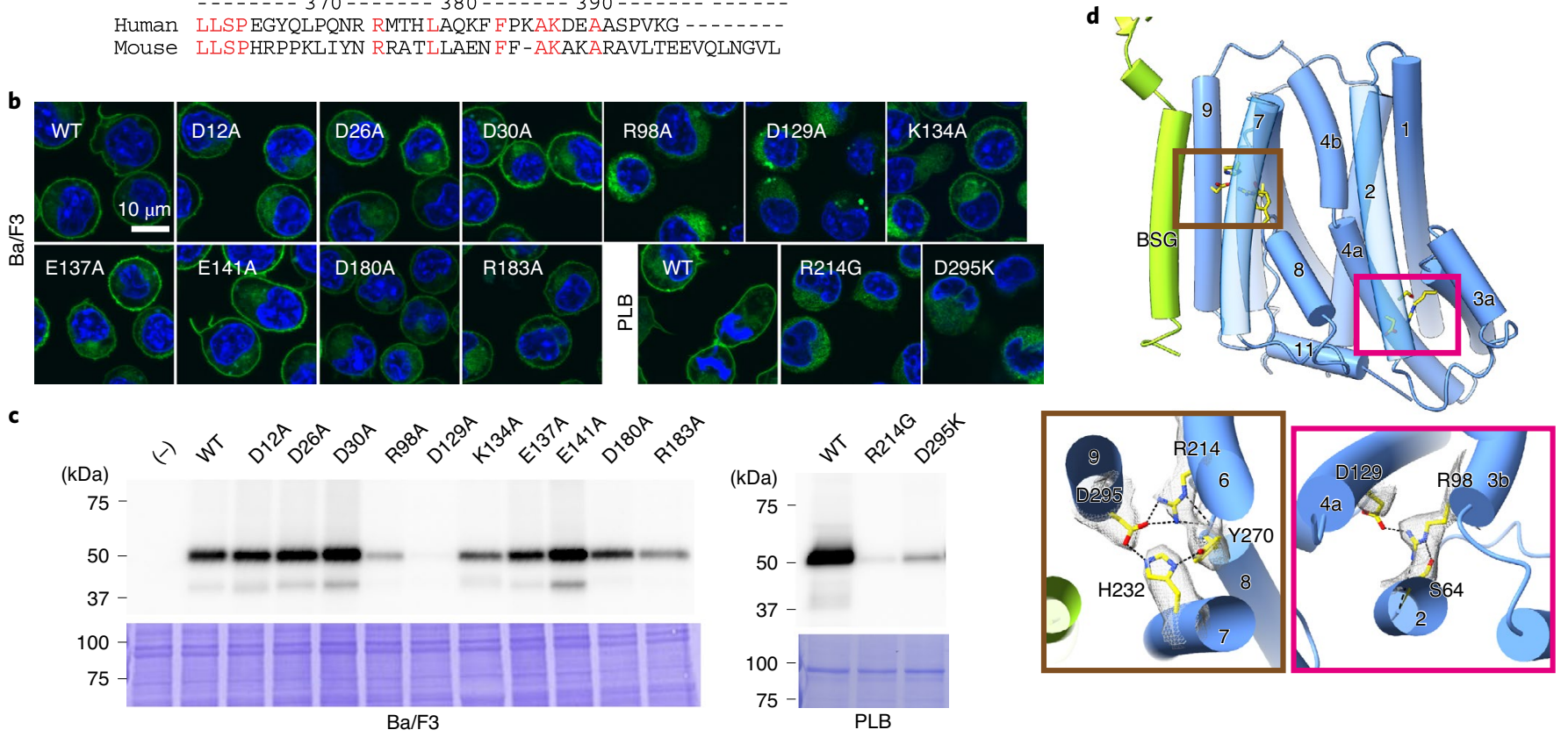

Fig. 3 | Charged residues in transmembrane helices of hXkr8. a, The sequences of hXkr8 (UniProt: Q9H6D3) and mXkr8 (UniProt: Q8C0T0) were analyzed using the MUSCLE Program (EMBL-EBI). Helices are shadowed and numbered. Conserved residues are in red. Negatively (Glu and Asp) and positively charged residues (Lys and Arg) in the lipid layer are highlighted in yellow and green, respectively. Residues (R42, Q155 and W309) that coordinate with the head group of PtdCho are highlighted in light blue. The amino acid residues mutated in Figs. 3-6 are indicated in the bottom line. b,c, GFP-tagged WT and indicated mutant mXkr8 (D12A, D26A, D30A, R98A, D129A, K134A, E137A, E141A, D180A and R183A) or hXkr8 (R214G and D295K) were stably expressed in mouse Ba/F3 or human PLB985, respectively, and observed under a fluorescent microscope (b). In c, whole-cell lysates (Ba/F3) or the light membrane fraction (PLB) were analyzed by western blotting with anti-GFP or stained by CBB. d, Stabilization of transmembrane $\alpha$-helices by salt bridges. The regions at which helices $\alpha 6-\alpha 9$ and helices $\alpha 4 a$ and $\alpha 3 b$ interact with each other are expanded with local density from the cryo-EM map (gray mesh). Source data for $\mathbf{b}$ and $\mathbf{c}$ are available online. CBB, Coomassie brilliant blue.

bottom inside the molecule (Fig. 4e; PDB 7DCE) and appeared to provide a path for scrambling phospholipids.

A hydrophobic cleft on the surface. The hXkr8-hBSG complex contains a hydrophobic cleft facing the lipid bilayer in the upper middle part of the complex (Fig. 5a,b). The aperture was mainly surrounded by $\alpha 2, \alpha 4, \alpha 6$ and $\alpha 7$. Tilted $\alpha 8$, as well as the loop between $\alpha 8$ and $\alpha 9$, supported the crack from below. We detected an excess density in this cleft, which was consistent with PtdCho (Fig. 5c), and an LC-MS/MS analysis of the complex confirmed its presence (Fig. 5d and Extended Data Fig. 9). Bound phospholipids were expected to originate from Sf9 cells because extra phospholipids were not added during protein preparation, indicating the strong affinity of $\mathrm{hXkr8}$ for phospholipids. The lipid fit well in the cleft (Fig. 5a,b). The contact area between hXkr8 and PtdCho, calculated by Protein Interfaces, Surfaces and Assemblies (PISA) ${ }^{38}$, was approximately $800 \AA^{2}$, which was similar to that observed between the PtdCho transfer protein (PCTP) and PtdCho (PDB 1 ln 2$)^{39}$. The head group of PtdCho fit well into the space comprising R42, W45, Q155 and W309 (Fig. 5e). The two acyl chains of PtdCho in the narrow cleft were linked by 12 hydrophobic amino acid residues (L44, L48, L52, L55, L140, L148, I152, V229, F233, L234, W237 and V263) (Fig. 5f). The arrangement of these amino acids with the lipid head group and acyl groups indicated that the aperture accepts other phospholipids.

We mutated three (LV-3A; L48, L148 and V229) or five (LVF-5A; L48, L52, L148, V229 and F233) hydrophobic amino acids in the middle of the aperture (Fig. 5f); R42, W45, Q155 and W310 (Fig. 5e) to alanine; C-terminally tagged them with GFP; and expressed them in $\mathrm{Ba} / \mathrm{F} 3$ cells. All mutants, except for LVF-5A, were expressed at plasma membrane; however, their expression levels differed (Fig. 5g). W310A and Q155A supported the exposure of PtdSer substantially less than wild-type Xkr8, whereas R42A and LV-3A lost the ability to expose PtdSer (Fig. 5h). These results suggest that in addition to stabilizing the complex, the hydrophobic cleft occupied by PtdCho contributed to scrambling phospholipids.

Constitutively active mutation. The W45A mutant was expressed as efficiently as wild-type $\mathrm{mXkr8}$, and localized to the plasma 
a

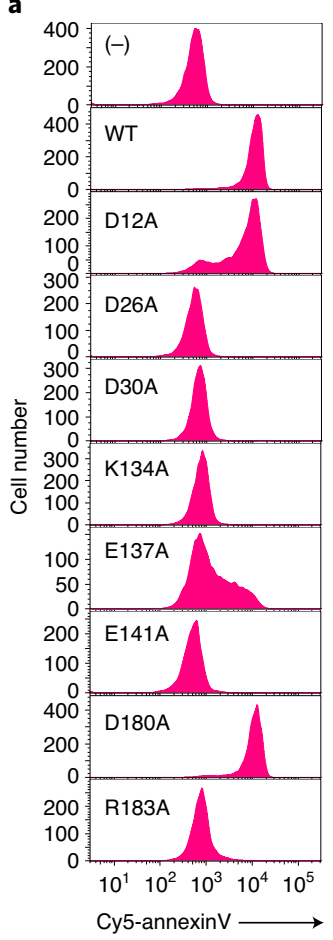

b
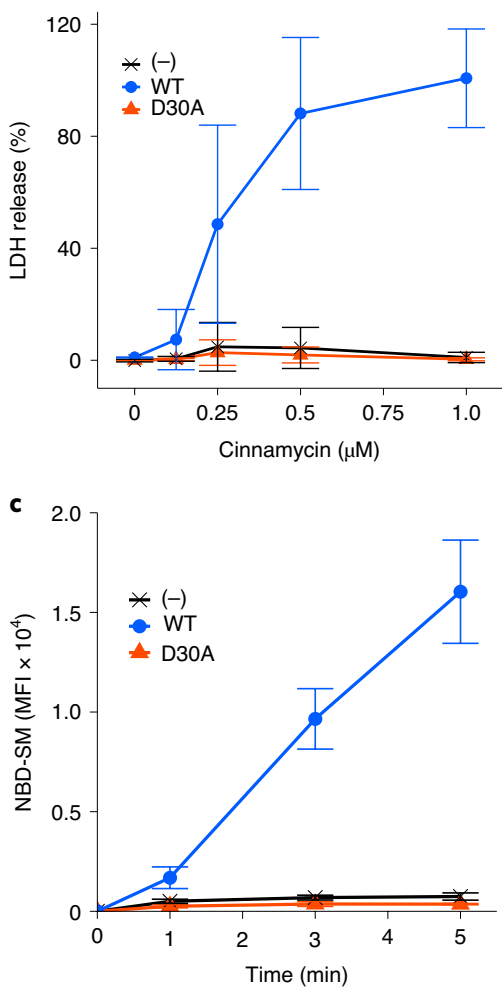

d

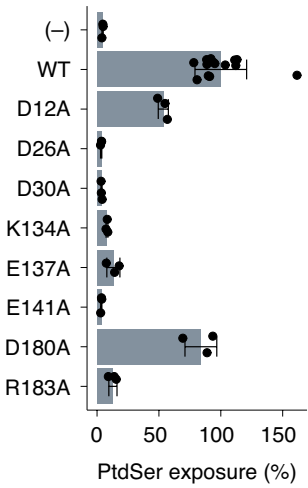

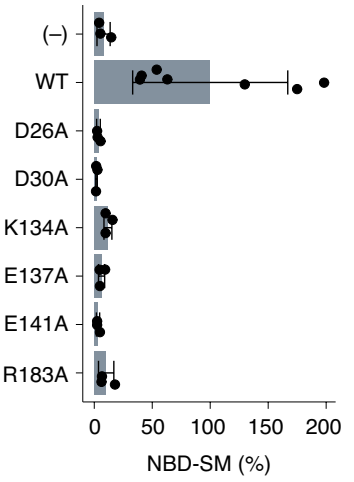

e
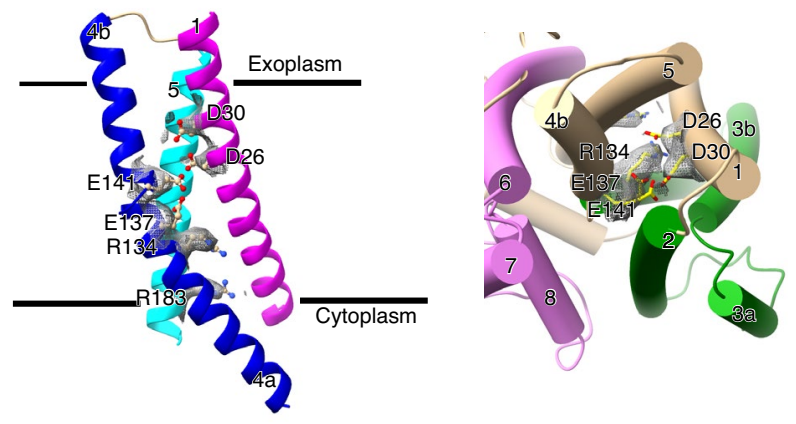

Fig. 4 | Requirement of charged residues in the membrane region of $m X k r 8$ to scramble phospholipids. $a, B a / F 3$ ( - ) and its transformants expressing the WT or indicated mutant $m X k r 8$ were stained with Cy5-annexin $V$ and analyzed by flow cytometry. $\mathbf{b}, \mathrm{Ba} / \mathrm{F} 3(-)$ ) and its transformants expressing WT or D30A mutant $\mathrm{mXkr} 8$ were incubated at $4^{\circ} \mathrm{C}$ for $15 \mathrm{~min}$ with the indicated concentrations of cinnamycin and the released LDH was quantified. Experiments were performed three times. The data, expressed as a percentage of that released with $1 \%$ Triton-X100, are presented as mean values \pm s.d. $\mathbf{c}$, Ba/F3 ( - ) and its transformants expressing WT or D30A mutant $\mathrm{mXkr} 8$ were incubated at $20^{\circ} \mathrm{C}$ with NBD-SM for the indicated periods. Incorporated NBD-SM was analyzed by flow cytometry, and its mean fluorescence intensity (MFI) was determined. Experiments were performed three times. Data are presented as mean values \pm s.d. d, The scramblase activity of $\mathrm{Ba} / \mathrm{F} 3$ transformants expressing WT or indicated mutant mXkr8 was assayed for the exposure of PtdSer (at $4{ }^{\circ} \mathrm{C}$ for $15 \mathrm{~min}$ ), sensitivity to cinnamycin $\left(0.5 \mu \mathrm{M}\right.$ )-induced $\mathrm{LDH}$ release (at $4{ }^{\circ} \mathrm{C}$ for $15 \mathrm{~min}$ ) and the incorporation of NBD-SM (at $20^{\circ} \mathrm{C}$ for $5 \mathrm{~min}$ ). Experiments were performed at least three times. Relative activities to that of WT are presented as mean values \pm s.d. e, Close-up side and top views of charged amino acids (Glu, Asp and Arg) in the $\alpha 1, \alpha 4$ and $\alpha 5$ helices. The side chains of charged amino acids are represented in a colored ball and stick model with the cryo-EM map superimposed (gray mesh). Source data for $\mathbf{b}$-d are available online.

membrane of $\mathrm{Ba} / \mathrm{F} 3$ cells (Fig. $5 \mathrm{~g}$ ). When we examined the exposure of PtdSer at $4{ }^{\circ} \mathrm{C}$, the W45A transformants exposed PtdSer more abundantly and uniformly than wild-type mXkr8 (Fig. 6a), suggesting that scrambling activity was enhanced by the W45A mutation. The Annexin V-binding assay was then performed at room temperature to examine whether enhanced scramblase activity overcomes the flippase activity that translocates PtdSer from the outer leaflet to the inner leaflet. As shown in Fig. 6b, a small cell population expressing wild-type $\mathrm{mXkr8}$ bound Annexin $\mathrm{V}$, whereas all the population of W45A transformants firmly bound it (Fig. 6b). Furthermore, a low concentration of cinnamycin efficiently lysed W45A-expressing cells at $37^{\circ} \mathrm{C}$ (Fig. $6 \mathrm{c}$ ), indicating W45A mutant Xkr8 scrambled PtdEtn to the cell surface against flippase activity to transport PtdEtn. The ability of W45A mutant mXkr8 to internalize $\mathrm{NBD}-\mathrm{SM}$ at $20^{\circ} \mathrm{C}$ was approximately threefold stronger than that of wild-type mXkr8 (Fig. $6 \mathrm{~d}$ ), confirming the enhanced scrambling activity of the W45A mutant.

mXkr8 is activated by phosphorylation ${ }^{16}$, and the mutation of three phosphorylation sites in its C-terminal tail region (3A; T356A/S361A/ T375A) markedly reduced its scrambling activity, which is consistent with previous findings ${ }^{16}$ (Fig. 6e,f). On the other hand, the transformants expressing the $3 \mathrm{~A}-\mathrm{W} 45 \mathrm{~A}$ mutant intensely exposed PtdSer, indicating that the W45A mutation provided $\mathrm{mXkr8}$ constitutively active or phosphorylation-independent scramblase. Similarly, the 3A mutation reduced sensitivity to cinnamycin, and the W45A mutation rescued this effect (Fig. 6f). The exposure of PtdSer and sensitivity to cinnamycin caused by the W45A mutation were entirely blocked by mutating D30 to alanine (3A-D30A/W45A) (Fig. 6e,f). These results indicate that $\mathrm{W} 45$ serves as an essential residue regulating the phospholipid-scrambling activity of Xkr8 at plasma membranes.

\section{Discussion}

A critical step in the translocation of phospholipids between the outer and inner leaflets of lipid bilayers is the movement of their hydrophilic head group across the hydrophobic core, and scramblase may provide a hydrophilic path(s) to facilitate this process ${ }^{23}$. Regarding TMEM16F of $\mathrm{Ca}^{2+}$-dependent scramblases and its homologs, various models have been proposed for scrambling phospholipids $^{24-27}$. The Xkr8-BSG complex has a cuboid-like structure with no similarities to TMEM16 family members or other proteins in the database. Salt bridges between hydrophilic amino acids connected several $\alpha$-helices in the middle of the transmembrane segments (BSG with $\alpha 9$; among $\alpha 6-\alpha 9$; $\alpha 3$ with $\alpha 4$ ). The critical residues for the interactions between $\alpha 6$ and $\alpha 9$ (R214 and D295) and between $\alpha 4 \mathrm{a}$ and $\alpha 3 \mathrm{~b}$ (D129 and R98) were well conserved among the XK family (Supplementary Fig. 3), suggesting that these members have 
a

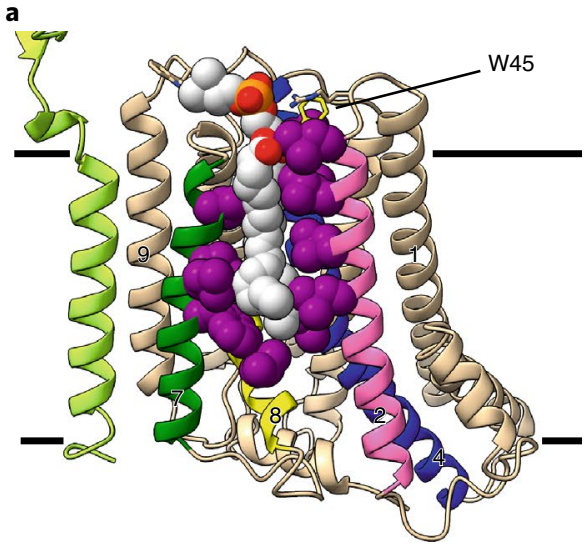

b

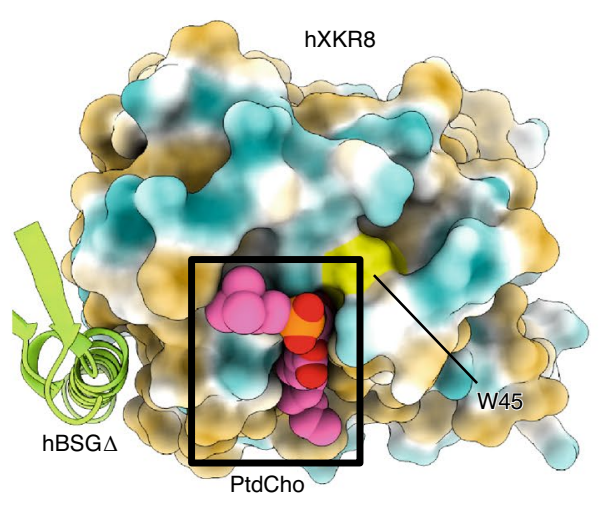

e

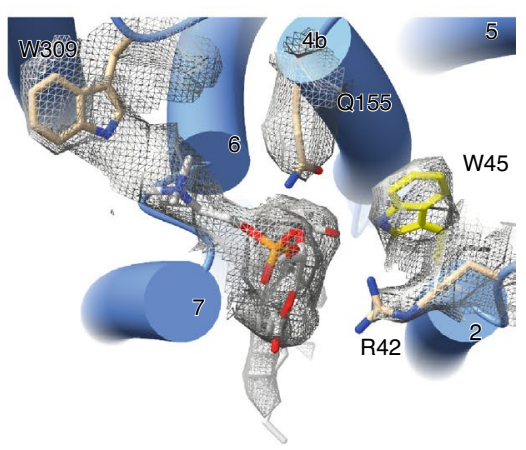

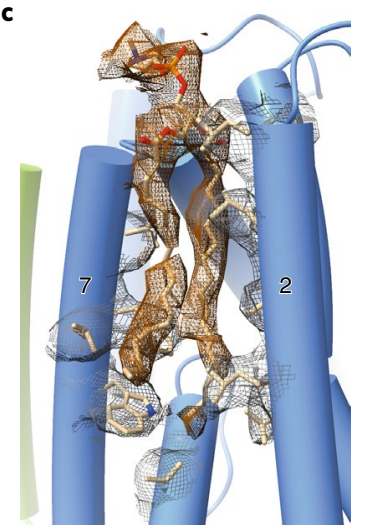

d
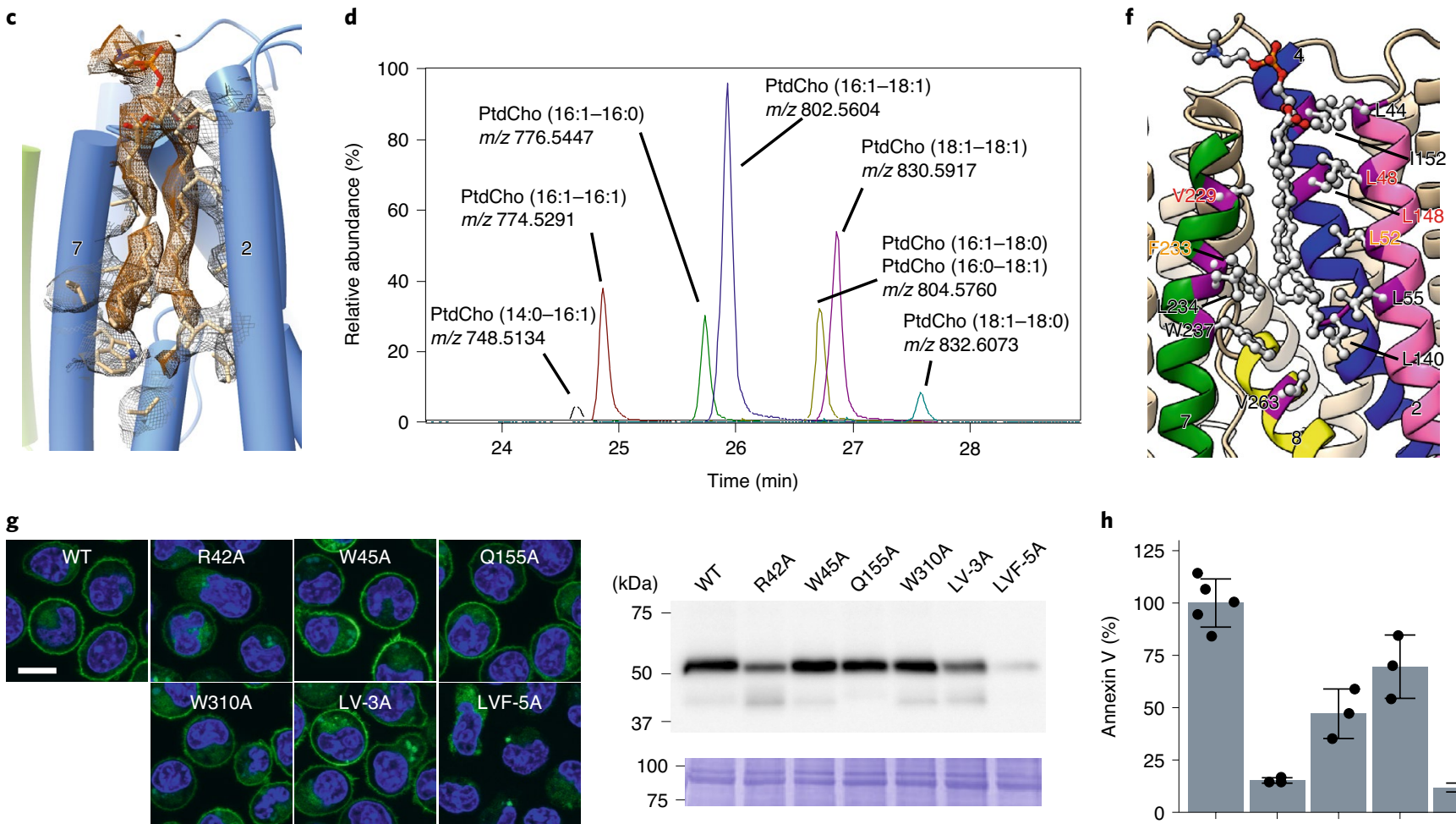

h

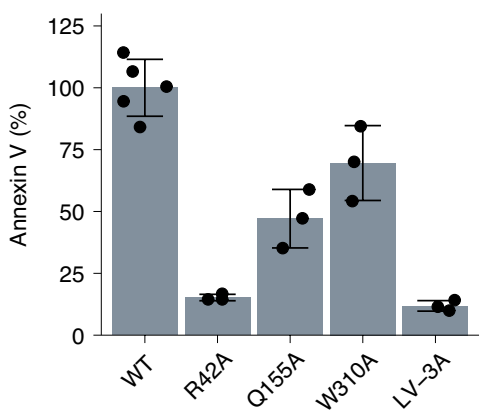

Fig. 5 | A lipid-binding cleft in hXkr8. a, Front view of the hXkr8-hBSG $\Delta$ complex. Hydrophobic residues surrounding PtdCho are in magenta spheres. PtdCho is in the silver sphere with the colored element. b. Top view of the complex. hXkr8 is in the colored surface view, brown, hydrophobic; blue, polar; white, neutral. PtdCho is colored in pink with oxygen in red and phosphorus in orange. c, A cryo-EM map of PtdCho (brown mesh) and surrounding residues (black mesh) is superimposed on the atomic model. d, LC-MS analysis of the lipid in the hXkr8-hBSG $\Delta$ complex in the negative ionization mode. The $m / z$ values corresponding to the formate adduct ions of PtdCho species are indicated. e, A close-up top view of PtdCho with residues (W309, Q155, W45 and R42) that coordinate with the head group of PtdCho. The side chains of these amino acids and PtdCho are in a stick model with the superimposed cryo-EM map (gray mesh). f, Hydrophobic residues (Leu, lle, Val, Phe and Trp) surrounding PtdCho are in a ball and stick structure. In the LV-3A mutant, L48, L148 and V229 were replaced by Ala (red). The LVF-5A mutant carries additional L52A and F233A mutations (orange). g, GFP-tagged WT and indicated mutant $\mathrm{mXkr8}$ were expressed in mouse $\mathrm{Ba} / \mathrm{F3}$, and observed under a fluorescent microscope. On the right, whole-cell lysates were analyzed by western blotting with anti-GFP or stained by CBB. Scale bar, $10 \mu \mathrm{m}$. $\mathbf{h}$, The scrambling activity of the indicated mutants was examined by incubating on ice for 15 min with Cy5-Annexin V. MFI obtained by each mutant was normalized by its expression level, and the percentage of that observed with WT mXkr8 was determined. Experiments were performed three times. The data are presented as mean values \pm s.d. Source data for $\mathbf{g}$ and $\mathbf{h}$ are available online.

a similar structure. In fact, Straub et al. ${ }^{42}$ recently reported the tertiary structure of rat Xkr9 with a similar fold as human Xkr8.

The amino acid sequence of Xkr8 is evolutionally well conserved among vertebrates, and a model predicted an almost identical structure of mXkr8 to that of hXkr8. Among ten members of the TMEM16 family, mouse lymphoma Ba/F3 cells only express TMEM16F as their plasma membrane $\mathrm{Ca}^{2+}$-dependent scramblase ${ }^{40}$.
$\mathrm{Ba} / \mathrm{F} 3$ cells lacking TMEM16F and Xkr8 genes do not expose PtdSer when stimulated with a $\mathrm{Ca}^{2+}$ ionophore or apoptosis inducer. We previously demonstrated that when this cell line was transformed by mXkr8, they exposed PtdSer at $4{ }^{\circ} \mathrm{C}$, but not at room temperature or higher $^{16}$. Although the scramblase activity of TMEM16F and Xkr8 is not markedly affected by temperature ${ }^{16,40,41}$, lowering the temperature inhibits P4-ATPase activity ${ }^{37}$. Since the kinase inhibitor 


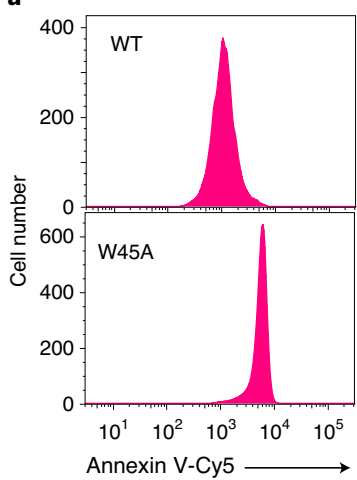

d

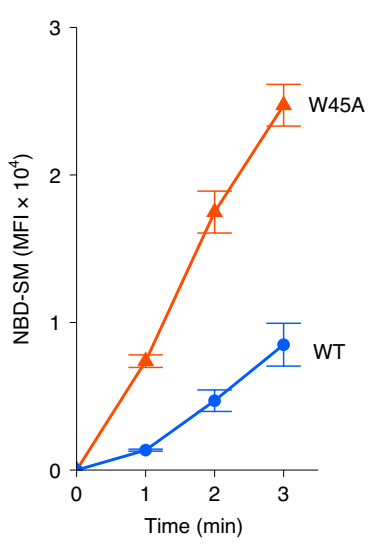

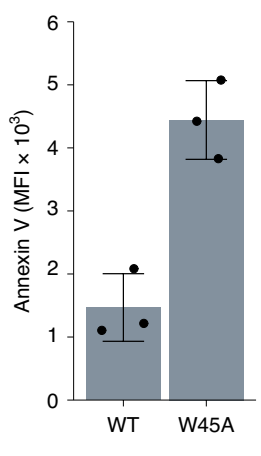

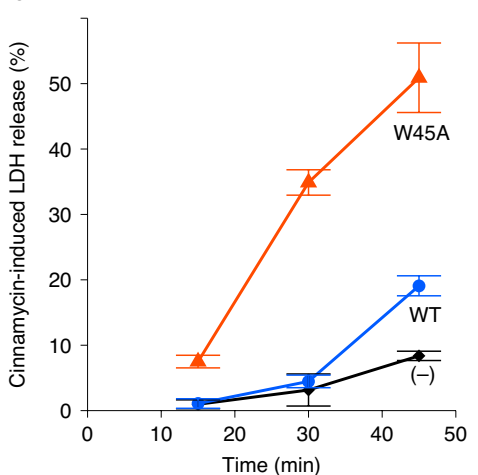

$\mathbf{f}$

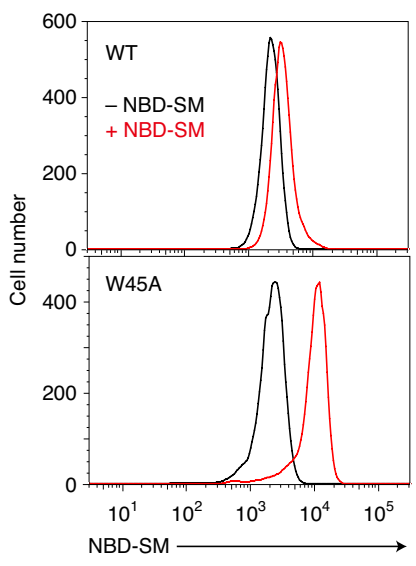

e

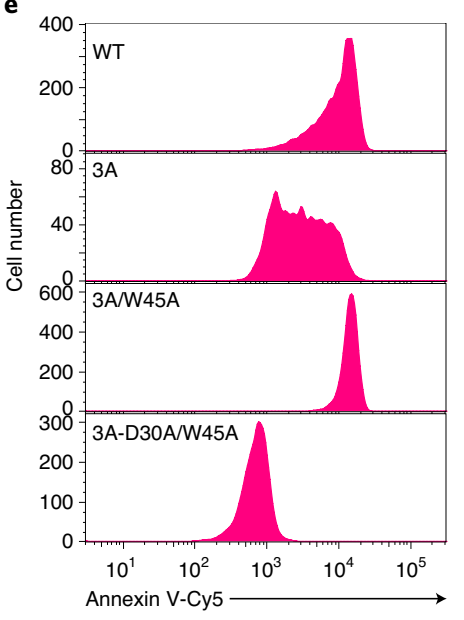

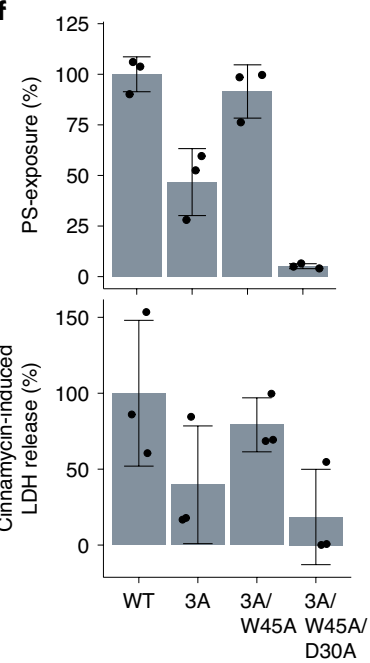

Fig. 6 | Regulation of phospholipid scrambling by a tryptophan at the extracellular end of the hydrophilic pathway. a,b, Effects of the W45A mutation on the exposure of PtdSer. Ba/F3 transformants expressing WT or W45A mutant mXkr8-GFP were subjected to Annexin $\mathrm{V}$ binding at $4{ }^{\circ} \mathrm{C}$ for $1 \mathrm{~min}(\mathbf{a})$ or at $20^{\circ} \mathrm{C}$ for $15 \mathrm{~min}$ (b). Experiments were performed three times. The MFI data are presented as mean values \pm s.d. $\mathbf{c}$, Effects of the W45A mutation on the exposure of PtdEtn. Parental Ba/F3 (-) and its transformants expressing WT or W45A mXkr8-GFP were treated with $0.0625 \mu \mathrm{M}$ cinnamycin at $37^{\circ} \mathrm{C}$ for the indicated period. $\mathrm{LDH}$ released into the buffer was quantified. Experiments were performed three times. Data are presented as mean values \pm s.d. $\mathbf{d}$, Effects of the W45A mutation on the incorporation of NBD-SM. Ba/F3 transformants expressing WT or W45A were incubated at $20^{\circ} \mathrm{C}$ for the indicated period, and analyzed by flow cytometry. Experiments were performed three times. The MFI data are presented as mean values \pm s.d. The right panels show representative fluorescence-activated cell sorting (FACS) profiles before and after the 1-min incubation with NBD-SM. e,f, Phosphorylation-independent scrambling by the W45A mutant mXkr8. Ba/F3 cells were transformed with the WT, 3A (T356A/S361A/T375A), 3A-W45A or 3A-W45A/D30A mutant of mXkr8-GFP; incubated with Cy5-Annexin V; and analyzed by flow cytometry (e). In f, scramblase activity in Ba/F3 transformants expressing WT or indicated mutant $\mathrm{mXkr} 8$ was assessed at $4{ }^{\circ} \mathrm{C}$ for the exposure of PtdSer (for $15 \mathrm{~min}$ ) and sensitivity to cinnamycin $(0.5 \mu \mathrm{M}$ )-induced LDH release (for $15 \mathrm{~min}$ ). Experiments were performed three times. Relative activities to that of the WT are presented as mean values \pm s.d. Source data for $\mathbf{a}-\mathbf{d}$ and $\mathbf{f}$ are available online.

blocks the exposure of PtdSer in $\mathrm{Ba} / \mathrm{F} 3$ transformants expressing $\mathrm{mXkr8}$, phosphorylation by intrinsic kinase(s) may activate $\mathrm{mXkr8}$ in $\mathrm{Ba} / \mathrm{F} 3$ cells ${ }^{16}$. Using this mouse system, we assayed the scramblase activity of mXkr8 mutants. mXkr8 mutants were stably expressed in $\mathrm{Ba} / \mathrm{F} 3$ cells deficient in TMEM16F and Xkr8 (ref. ${ }^{16}$ ), and scramblase activity for the exposure of PtdSer or PtdEtn was measured at $4^{\circ} \mathrm{C}$ to inhibit flippase. The internalization of NBD-SM, which was not expected to be affected by the flippases ${ }^{37}$, was performed at a higher temperature. The replacement of a tryptophan residue (W45) located at the extracellular boundary to alanine made the molecule constitutively active or phosphorylation-independent, suggesting that the active form of the mXkr8-BSG complex has a similar structure to that elucidated in the present study. This may agree with the report that caspase cleavage does not induce a substantial conformational change in rat Xkr9 (ref. ${ }^{42}$ ).

We detected a hydrophobic aperture occupied by a single PtdCho molecule on one surface of hXkr8 facing the outer leaflet of the lipid bilayer. The alanine mutations of three hydrophobic residues
(L48, L148 and V229) surrounding the PtdCho blocked the phospholipid scrambling evoked by the mXkr8-BSG complex. The mutations of the residues (R42 and Q155) that coordinate to the head group of PtdCho also affected the scrambling activity. A series of eight charged residues were present in the helixes of the internal side of the molecule, and at least six of them were indispensable for scrambling phospholipids, thereby supporting their provision of a pathway for phospholipid scrambling. These mutations, except for R183A in the exposure of PtdEtn, similarly affected the outward (PtdSer and PtdEtn exposure) and inward translocation (the incorporation of NBD-SM) of phospholipids, indicating that different phospholipids use the same or overlapping paths for inward and outward movements in most cases.

Phospholipid scrambling is a downhill reaction that does not require ATP and serves to destroy the asymmetrical distribution of phospholipids between two lipid layers ${ }^{2}$. Based on the structure of hXkr8-BSG and the results obtained from the mutational analysis of $\mathrm{mXkr8-BSG}$, we propose the following working models for the 
activation mechanism of Xkr8 to scramble phospholipids (Extended Data Fig. 10). In the first model, phospholipids, such as PtdCho and sphingomyelin, in the outer layer of plasma membranes are recruited to the hydrophobic cleft of Xkr8-BSG. Caspase 3 activated during apoptosis cleaves $\alpha 11$ to remove the C-terminal tail region, or a putative kinase(s) phosphorylates residues at the C-terminal tail region ${ }^{16}$. The W45A mutant works as a constitutive-active form, suggesting that the caspase cleavage may cause the bending of W45. The phospholipid in the aperture then approaches through $\alpha 2$ and $\alpha 4 b$ to the scrambling path via an interaction with R158, Q163 and Q145. As proposed for the scrambling mechanism of TMEM16 and opsin ${ }^{43-46}$, once the phospholipid enters the trail, it is likely to affect or modify the pathway. The essential role of acidic residues (D26, D30, E137 and E141) for the scrambling activity suggests that the repulsion force generated between the negative charge of the phospholipid's head group and acidic residues in the path may widen the track to allow the phospholipid to pass. The $\alpha$-helixes $(\alpha 1, \alpha 2$, $\alpha 4$ and $\alpha 5)$ composing the hydrophilic pathway carry many hydrophobic residues between the helixes which may allow acyl groups to move through the molecule. The mutation of K134 (corresponding to R134 in human Xkr8) and R183 prevented the scrambling, suggesting that phospholipids that travel to the cellular side are recruited by R134 and R183.

In another model (Model 2), the activation of the Xkr8-BSG complex by caspase may broaden the space between $\alpha 1$ and $\alpha 5$, and phospholipids scramble according to the 'credit card' model ${ }^{23}$ through this area by inserting their charged head group into the hydrophilic cleft, leaving the acyl group into the lipid layer. In this model, phospholipids do not use the hydrophobic cleft as the entry site, and PtdCho serves to stabilize the molecule. The acidic residues (D26, D30, E137 and E141) in the hydrophilic cleft are unlikely to be used as stepping stones for the head group of phospholipids, and they may be used to widen the path. Although we prefer the first model, further studies on the structures of different intermediate states carrying the phospholipids will be needed to prove this model. As described above, Xkr8 is a member of the XK family, which comprises nine members in humans (Supplementary Fig. 3). The tertiary structures of human Xkr8-BSG complex and rat Xkr9 (ref. ${ }^{42}$ ) will provide a template for understanding the molecular mechanisms underlying phospholipid scrambling and structure-function analyses of the XK family.

\section{Online content}

Any methods, additional references, Nature Research reporting summaries, source data, extended data, supplementary information, acknowledgements, peer review information; details of author contributions and competing interests; and statements of data and code availability are available at https://doi.org/10.1038/ s41594-021-00665-8.

Received: 13 March 2021; Accepted: 19 August 2021; Published online: 8 October 2021

\section{References}

1. Leventis, P. A. \& Grinstein, S. The distribution and function of phosphatidylserine in cellular membranes. Annu. Rev. Biophys. 39, 407-427 (2010).

2. Bevers, E. M. \& Williamson, P. L. Getting to the outer leaflet: physiology of phosphatidylserine exposure at the plasma membrane. Physiol. Rev. 96, 605-645 (2016).

3. Birge, R. B. et al. Phosphatidylserine is a global immunosuppressive signal in efferocytosis, infectious disease, and cancer. Cell Death Differ. 23, 962-978 (2016).

4. Segawa, K. \& Nagata, S. An apoptotic 'eat me' signal: phosphatidylserine exposure. Trends Cell Biol. 25, 649-650 (2015).

5. Nagata, S., Sakuragi, T. \& Segawa, K. Flippase and scramblase for phosphatidylserine exposure. Curr. Opin. Immunol. 62, 31-38 (2020).
6. Segawa, K. et al. Caspase-mediated cleavage of phospholipid flippase for apoptotic phosphatidylserine exposure. Science 344, 1164-1168 (2014).

7. Kornberg, R. D. \& McConnell, H. M. Lateral diffusion of phospholipids in a vesicle membrane. Proc. Natl Acad. Sci. USA 68, 2564-2568 (1971).

8. Suzuki, J., Umeda, M., Sims, P. J. \& Nagata, S. Calcium-dependent phospholipid scrambling by TMEM16F. Nature 468, 834-838 (2010).

9. Suzuki, J., Denning, D. P., Imanishi, E., Horvitz, H. R. \& Nagata, S. Xk-related protein 8 and CED-8 promote phosphatidylserine exposure in apoptotic cells. Science 341, 403-406 (2013).

10. Suzuki, J. et al. Calcium-dependent phospholipid scramblase activity of TMEM16 protein family members. J. Biol. Chem. 288, 13305-13316 (2013).

11. Bushell, S. R. et al. The structural basis of lipid scrambling and inactivation in the endoplasmic reticulum scramblase TMEM16K. Nat. Commun. 10, 3956 (2019).

12. Brunner, J. D., Lim, N. K., Schenck, S., Duerst, A. \& Dutzler, R. X-ray structure of a calcium-activated TMEM16 lipid scramblase. Nature 516, 207-212 (2014).

13. Malvezzi, M. et al. $\mathrm{Ca}^{2+}$-dependent phospholipid scrambling by a reconstituted TMEM16 ion channel. Nat. Commun. 4, 2367 (2013).

14. Pelz, T. et al. An ancestral TMEM16 homolog from Dictyostelium discoideum forms a scramblase. PLoS ONE 13, e0191219-e0191222 (2018).

15. Suzuki, J., Imanishi, E. \& Nagata, S. Xkr8 phospholipid scrambling complex in apoptotic phosphatidylserine exposure. Proc. Natl Acad. Sci. USA 113, 9509-9514 (2016).

16. Sakuragi, T., Kosako, H. \& Nagata, S. Phosphorylation-mediated activation of mouse Xkr8 scramblase for phosphatidylserine exposure. Proc. Natl Acad. Sci. USA 33, 2907-2912 (2019).

17. Yamashita, Y., Suzuki, C., Uchiyama, Y. \& Nagata, S. Infertility caused by inefficient apoptotic germ cell clearance in Xkr8-deficient male mice. Mol. Cell. Biol. 40, e00402-e00419 (2020).

18. Kawano, M. \& Nagata, S. Lupus-like autoimmune disease caused by a lack of Xkr8, a caspase-dependent phospholipid scramblase. Proc. Natl Acad. Sci. USA 280, 2132-2137 (2018).

19. Alvadia, C. et al. Cryo-EM structures and functional characterization of the murine lipid scramblase TMEM16F. eLife 8, 213 (2019).

20. Timcenko, M. et al. Structure and autoregulation of a P4-ATPase lipid flippase. Nature 571, 366-370 (2019).

21. Nakanishi, H. et al. Crystal structure of a human plasma membrane phospholipid flippase. J. Biol. Chem. 295, 10180-10194 (2020).

22. Hiraizumi, M., Yamashita, K., Nishizawa, T. \& Nureki, O. Cryo-EM structures capture the transport cycle of the P4-ATPase flippase. Science 365, 1149-1155 (2019).

23. Pomorski, T. \& Menon, A. K. Lipid flippases and their biological functions. Cell. Mol. Life Sci. 63, 2908-2921 (2006).

24. Malvezzi, M. et al. Out-of-the-groove transport of lipids by TMEM16 and GPCR scramblases. Proc. Natl Acad. Sci. USA 7, 201806721-201806710 (2018).

25. Falzone, M. E. et al. Structural basis of $\mathrm{Ca}^{2+}$-dependent activation and lipid transport by a TMEM16 scramblase. eLife 8, e43229 (2019).

26. Bethel, N. P. \& Grabe, M. Atomistic insight into lipid translocation by a TMEM16 scramblase. Proc. Natl Acad. Sci. USA 113, 14049-14054 (2016).

27. Feng, S. et al. Cryo-EM studies of TMEM16F calcium-activated ion channel suggest features important for lipid scrambling. Cell Rep. 28, 567-579.e564 (2019).

28. Hunte, C. \& Michel, H. Crystallisation of membrane proteins mediated by antibody fragments. Curr. Opin. Struct. Biol. 12, 503-508 (2002).

29. Hauer, F. et al. GraDeR: membrane protein complex preparation for single-particle cryo-EM. Structure 23, 1769-1775 (2015).

30. Killian, J. A. \& von Heijne, G. How proteins adapt to a membrane-water interface. Trends Biochem. Sci. 25, 429-434 (2000).

31. Norimatsu, Y., Hasegawa, K., Shimizu, N. \& Toyoshima, C. Proteinphospholipid interplay revealed with crystals of a calcium pump. Nature 545, 193-198 (2017).

32. Krissinel, E. \& Henrick, K. Secondary-structure matching (SSM), a new tool for fast protein structure alignment in three dimensions. Acta Crystallogr. D Biol. Crystallogr. D60, 2256-2268 (2004).

33. Webb, B. \& Sali, A. Comparative protein structure modeling using MODELLER. Curr. Protoc. Bioinform. 54, 5.6.1-5.6.37 (2016).

34. Jung, H. H. et al. McLeod phenotype associated with a XK missense mutation without hematologic, neuromuscular, or cerebral involvement. Transfusion 43, 928-938 (2003).

35. Russo, D. C. W., Lee, S., Reid, M. E. \& Redman, C. M. Point mutations causing the McLeod phenotype. Transfusion 42, 287-293 (2002).

36. Makino, A. et al. Cinnamycin (Ro 09-0198) promotes cell binding and toxicity by inducing transbilayer lipid movement. J. Biol. Chem. 278, 3204-3209 (2003)

37. Segawa, K., Kurata, S. \& Nagata, S. Human type IV P-type ATPases that work as plasma membrane phospholipid flippases, and their regulation by caspase and calcium. J. Biol. Chem. 291, 762-772 (2016). 
38. Krissinel, E. \& Henrick, K. Inference of macromolecular assemblies from crystalline state. J. Mol. Biol. 372, 774-797 (2007).

39. Roderick, S. L. et al. Structure of human phosphatidylcholine transfer protein in complex with its ligand. Nat. Struct. Biol. 9, 507-511 (2002).

40. Gyobu, S., Ishihara, K., Suzuki, J., Segawa, K. \& Nagata, S. Characterization of the scrambling domain of the TMEM16 family. Proc. Natl Acad. Sci. USA 114, 6274-6279 (2017).

41. Watanabe, R., Sakuragi, T., Noji, H. \& Nagata, S. Single-molecule analysis of phospholipid scrambling by TMEM16F. Proc. Natl Acad. Sci. USA 115, 3066-3071 (2018).

42. Straub, M. S., Alvadia, C., Sawicka, M. \& Dutzler, R. Cryo-EM structures of the caspase activated protein XKR9 involved in apoptotic lipid scrambling. eLife 10, e69800 (2021).

43. Khelashvili, G. et al. Membrane lipids are both the substrates and a mechanistically responsive environment of TMEM16 scramblase proteins. J. Comput. Chem. 41, 538-551 (2020).

44. Morra, G. et al. Mechanisms of lipid scrambling by the $\mathrm{G}$ protein-coupled receptor opsin. Structure 26, 356-367 (2018)

45. Lee, B.-C. et al. Gating mechanism of the extracellular entry to the lipid pathway in a TMEM16 scramblase. Nat. Commun. 9, 3251 (2018).
46. Khelashvili, G. et al. Dynamic modulation of the lipid translocation groove generates a conductive ion channel in $\mathrm{Ca}^{2+}$-bound nhTMEM16. Nat. Commun. 10, 4972 (2019).

47. Yu, X.-L. et al. Crystal structure of HAb18G/CD147: implications for immunoglobulin superfamily homophilic adhesion. J. Biol. Chem. 283, 18056-18065 (2008)

Publisher's note Springer Nature remains neutral with regard to jurisdictional claims in published maps and institutional affiliations.

\section{(c) (i) Open Access This article is licensed under a Creative Commons \\ Attribution 4.0 International License, which permits use, sharing, adap- tation, distribution and reproduction in any medium or format, as long} as you give appropriate credit to the original author(s) and the source, provide a link to the Creative Commons license, and indicate if changes were made. The images or other third party material in this article are included in the article's Creative Commons license, unless indicated otherwise in a credit line to the material. If material is not included in the article's Creative Commons license and your intended use is not permitted by statutory regulation or exceeds the permitted use, you will need to obtain permission directly from the copyright holder. To view a copy of this license, visit http://creativecommons. org/licenses/by/4.0/.

(c) The Author(s) 2021 


\section{Methods}

Cell lines, recombinant proteins and materials. Spodoptera frugiperda 9 (Sf9) cells (ATCC CRL-1711) were cultured in Sf-900IIISFM (Gibco); PLB985 cells, EL-4 cells (ATCC TIB-39) and $B S G^{-/-} N P T N^{-/-}$W3 cells ${ }^{15}$ in RPMI-10\% FCS and $50 \mu \mathrm{M} \beta$-mercaptoethanol; TMEM16F $\mathrm{F}^{-/-} \mathrm{Xkr}^{-/-} \mathrm{Ba} / \mathrm{F} 3$ cells (ref. ${ }^{16}$ ) in RPMI- $10 \%$ FCS and 45 units per ml of IL-3; and HEK293T cells in DMEM-10\% FCS.

HRP-rabbit anti-GFP antibody (anti-GFP pAb-HRP-DirecT) and HRP-mouse anti-HA monoclonal antibody (Direct-Blot HRP anti-HA.11 Epitope Tag antibody) were from MBL and BioLegend, respectively. Biotin-conjugated Ro 09-0198 (ref. ${ }^{36}$ ) was a gift from K. Nagao (Kyoto University). Cy5-Annexin V was from BioVision. 1,2-dioleoyl-sn-glycero-3-phosphocholine (DOPC) was from Avanti Polar Lipids. NBD-SM was from Cayman CHEMICAL. LMNG, glycol-diosgenin (GDN), octaethyleneglycol mono- $n$-dodecylether $\left(\mathrm{C}_{12} \mathrm{E}_{8}\right)$ and fluorinated Fos-choline 8 (FC8) were from Anatrace. Cholesteryl hemisuccinate (CHS) and tobacco etch virus (TEV) protease were from Sigma-Aldrich.

Complementary DNAs and expression plasmids. The DNA sequence for hXkr8 (UniProt: Q9H6D3) was custom-designed to enhance messenger RNA stability and translational efficiency and synthesized by Thermo Fisher Scientific. hXkr8 cDNA was introduced into pBAC-3bV, a derivative of pFastBacl (Thermo Fisher Scientific), to express the protein tagged C-terminally with a TEV cleavage sequence, eight histidine residues, a c-Myc, eight histidine residues and monomeric EGFP (A206K) in this order ${ }^{48}$. This construct is hereafter referred to as hXkr8TH-mGFP. cDNAs for hBSG, mBSG, mXkr8 and mXkr8 S/T-3A (T356A/ S361A/T375A) mutant were described previously $y^{15,16}$. hBSG cDNA lacking the immunoglobulin-1 (Ig1) (amino acids 23-102) was produced by PCR, fused to a FLAG at the $\mathrm{C}$ terminus and introduced into $\mathrm{pBAC}-3 \mathrm{bV}$. The DNA fragments for LV-3A (L48A, L148A, V229A) and LVF-5A (L48, L52, L148, V229, F233) of mXkr8 were custom-synthesized by Eurofins Genomics. Other mutants of hBSG, hXkr8 and $\mathrm{mXkr8}$ were prepared by PCR using primers described in Supplementary Table 1. The resultant cDNAs were introduced into pMXs-Puro c-GFP', pCX4-bsr c-HA ${ }^{15}$, pPEF-mEGFP-FLAG ${ }^{16}$ or pNEF-HA (pEF-BOS-EX ${ }^{49}$ carrying a neomycin resistance gene and HA tag). The authenticity of cDNAs was confirmed by sequencing.

Purification of hXkr8-hBSG $\Delta$ complex. Baculovirus carrying hXkr8-THmGFP or hBSG $\Delta$ cDNA was produced using Bac-to-Bac Baculovirus Expression System (Thermo Fisher Scientific). Sf9 cells were co-infected with baculoviruses carrying hXkr8-TH-mGFP or hBSG $\Delta$, and cultured for $60 \mathrm{~h}$. Infected Sf9 cells were suspended in $20 \mathrm{mM}$ HEPES-NaOH buffer ( $\mathrm{pH} 7.0$ ) containing $150 \mathrm{mM}$ $\mathrm{NaCl}, 1 \mathrm{mM}$ EDTA, $1 \mathrm{mM}$ EGTA, $0.4 \mathrm{mM}$ Pefabloc SC, $1 \mathrm{mM}$ pAPMSF, $1 \mathrm{mM}$ Tris(2-carboxyethyl)phosphine (TCEP) and protease inhibitors (cOmplete, EDTA-free, Roche Diagnostics). After adding 1.3 units per ml of benzonase (Merck), cells were disrupted on ice using an ultrasonic disrupter (Sonifier 450 Advanced; Branson) (0.5-s sonication at a level of 4 and 0.5-s cooling intervals for a total sonication period of $2.5 \mathrm{~min}$ ). Unbroken cells and nuclei were removed by centrifugation at $6,000 \mathrm{~g}$ for $25 \mathrm{~min}$, and crude membrane fractions were collected by centrifugation at $99,650 \mathrm{~g}$ for $1 \mathrm{~h}$. Membranes were homogenized using a Dounce homogenizer in $20 \mathrm{mM}$ HEPES-NaOH buffer ( $\mathrm{pH} 7.0$ ) containing $150 \mathrm{mM}$ $\mathrm{NaCl}, 25 \%$ glycerol, $50 \mathrm{mM}$ imidazole, $0.4 \mathrm{mM}$ Pefabloc SC, $1 \mathrm{mM}$ pAPMSF, $1 \mathrm{mM}$ TCEP and protease inhibitors, and subjected to sonication (0.5-s flash with $0.5-\mathrm{s}$ cooling; total sonication time of $30 \mathrm{~s}$ ) at an output level of 3. LMNG and CHS were added to $1.0 \%$ and $0.1 \%$, and incubated at $4{ }^{\circ} \mathrm{C}$ for $2 \mathrm{~h}$. After centrifugation at $99,650 \mathrm{~g}$ for $1 \mathrm{~h}$, the lysates were loaded onto a HisTrap High Performance column (Cytiva) attached to an HPLC system (Prominence; Shimadzu), and washed with 60-80 mM imidazole in Buffer A (20 mM HEPES-NaOH buffer ( $\mathrm{pH} 7.0$ ), $0.01 \%$ LMNG, $0.001 \%$ CHS, $150 \mathrm{mM} \mathrm{NaCl}$ and $1 \mathrm{mM} \mathrm{TCEP)} \mathrm{containing} 25 \%$ glycerol. The hXkr8-TH-mGFP/hBSG $\Delta$ complex was eluted with a linear $80-400 \mathrm{mM}$ imidazole gradient in Buffer A containing $25 \%$ glycerol and treated at $4{ }^{\circ} \mathrm{C}$ with TEV protease for $12-18 \mathrm{~h}$ while dialyzing against Buffer A containing $10 \%$ glycerol and $1 \mathrm{mM}$ EDTA. The sample was re-loaded over a HisTrap column equilibrated with $20 \mathrm{mM}$ HEPES-NaOH buffer (pH 7.0), 0.005\% LMNG, 0.0005\% CHS, $150 \mathrm{mM} \mathrm{NaCl}, 10 \%$ glycerol and $1 \mathrm{mM}$ TCEP, and the flowed-through fractions were concentrated approximately 100 -fold by ultrafiltration using an Amicon Ultra-15 50K filter.

Analytical size-exclusion chromatography was performed at $4{ }^{\circ} \mathrm{C}$ with a Superose 6 Increase 10/300 GL column (Cytiva) attached to an HPLC system. The column was developed with TBS (20 mM Tris- $\mathrm{HCl}(\mathrm{pH} 7.5)$ and $150 \mathrm{mM}$ $\mathrm{NaCl}$ ) containing $0.005 \%$ LMNG, $0.0005 \%$ CHS, $10 \%$ glycerol, $1 \mathrm{mM} \mathrm{DTT}, 10 \mu \mathrm{M}$ pAPMSF and $100 \mu \mathrm{M}$ Pefablock SC at a flow rate of $0.28 \mathrm{ml} \mathrm{min}^{-1}$. Proteins were detected using absorbance at $280 \mathrm{~nm}$.

Monoclonal antibodies against the hXkr8-hBSG complex. Rabbit monoclonal antibodies against hXkr8-hBSG $\Delta$ were established as described ${ }^{50}$. New Zealand White rabbits from KITAYAMA LABES were immunized with hXkr8-hBSG $\Delta$ using TiterMax (Sigma-Aldrich) or aluminum salts as adjuvants. IgG-positive B cells from peripheral mononuclear cells and splenocytes were cultured at $37^{\circ} \mathrm{C}$ for $11 \mathrm{~d}$ at 2 cells per well together with $2.5 \times 10^{4}$ Mitomycin C-treated murine EL-4 cells. Screening using the Octet Red96 System (ForteBio) identified two clones
(XBA14 and XBA18) that recognized hXkr8-hBSG $\Delta$. Their amino acid sequences differed at four positions at the variable region. However, their CDR3 domain had the same sequences, suggesting that they recognize the same epitope. DNA fragments for the variable regions of the heavy and light chains were inserted into pEF-BOS-based expression vectors carrying the constant region of human IgG heavy or light chain. The recombinant IgG (XBA14 and XBA18) was produced in FreeStyle 293-F cells (Thermo Fisher Scientific), and purified by Protein A-Sepharose (Cytiva) or MabSelect SuRe (Cytiva).

All animal studies were approved by the Animal Care and Use Committees of the Research Institute of Microbial Diseases, Osaka University and Chugai Pharmaceutical Co.

Preparation of the hXkr8-hBSG $\Delta-F a b$ complex. To prepare the Fab fragment, IgG was digested at $35^{\circ} \mathrm{C}$ for $2 \mathrm{~h}$ with Lys-C in Tris-HCl buffer ( $\mathrm{pH} 8.0$ ), and Fc fragments were removed using HiTrap SP HP (Cytiva) and MabSelect SuRe columns. Fab fragments (Fab14 and Fab18) were purified by gel filtration (Superdex $200 \mathrm{pg} 16 / 600$, Cytiva) in $20 \mathrm{mM}$ HEPES-NaOH (pH 7.1) containing $100 \mathrm{mM} \mathrm{NaCl}$. The hXkr8-hBSG $\Delta$ was then incubated with the Fab at $4^{\circ} \mathrm{C}$ for $20-30 \mathrm{~h}$ in $16 \mathrm{mM}$ Tris- $\mathrm{HCl}$ ( $\mathrm{pH} 7.5$ ), $4 \mathrm{mM}$ HEPES- $\mathrm{NaOH}$ (pH 7.1), $140 \mathrm{mM} \mathrm{NaCl}$ and $8 \%$ glycerol, and subjected to gel filtration using Superose 6 Increase 10/300 GL column (Cytiva) equilibrated with TBS containing $10 \%$ glycerol. Fractions containing hXkr8-hBSG $\Delta$-Fab were collected and concentrated by ultrafiltration using Vivaspin 2-100K (Cytiva).

In the cryo-EM analysis, detergents in the sample were removed by GraDeR as described ${ }^{29}$. The hXkr8-hBSG $\Delta$ was incubated with the Fab 18 at $4^{\circ} \mathrm{C}$ for $20-30 \mathrm{~h}$ in $20 \mathrm{mM}$ HEPES-NaOH buffer (pH 7.0), $136 \mathrm{mM} \mathrm{NaCl}, 7 \%$ glycerol and $0.7 \mathrm{mM}$ TCEP. Using a Gradient Master (BIO COMP), a linear gradient of glycerol (10\% to $30 \%)$ with a reverse gradient of detergents $(0.00225 \%$ to $0 \%$ for LNMG and $0.00075 \%$ to $0 \%$ for GDN) was established in $4 \mathrm{ml}$ of $20 \mathrm{mM}$ HEPES-NaOH buffer (pH 7.0) containing $100 \mathrm{mM} \mathrm{NaCl}, 1 \mathrm{mM}$ EGTA, $1 \mathrm{mM}$ TCEP, $10 \mu \mathrm{M} p$ APMSF and $100 \mu \mathrm{M}$ Pefabloc SC. Protein samples $(0.1-0.2 \mathrm{ml})$ were loaded on the top of the gradient, and centrifuged at $200,614 \mathrm{~g}$ for $15 \mathrm{~h}$ using a Beckman SW55Ti rotor. Gradients were fractionated using the Piston Gradient Fractionator (BIO COMP), and fractions carrying hXkr8-hBSG $\Delta$-Fab18 were pooled and concentrated by ultrafiltration using a Vivaspin 2-100K. Glycerol was removed by repeating concentration and 30-40-fold dilution with the grid buffer $(20 \mathrm{mM}$ HEPES-NaOH (pH 7.0), $100 \mathrm{mM} \mathrm{NaCl}, 1 \mathrm{mM}$ EGTA, $1 \mathrm{mM}$ TCEP, $10 \mu \mathrm{M}$ pAPMSF and $100 \mu \mathrm{M}$ Pefabloc SC) three times.

Crystallization and structural analysis. For crystallization of Fab14, $100 \mathrm{nl}$ of $7.1 \mathrm{mg} \mathrm{ml}^{-1}$ Fab14 was mixed with an equal volume of reservoir solution $(0.1 \%$ $\mathrm{n}$-Octyl- $\beta$-D-glucoside, $0.1 \mathrm{M}$ sodium citrate tribasic dihydrate and 22\% PEG3350 (Hampton)) and kept at $20^{\circ} \mathrm{C}$. Crystals were collected after $10 \mathrm{~d}$; soaked in $20 \mathrm{mM}$ HEPES-NaOH buffer ( $\mathrm{pH}$ 7.1) containing $0.1 \% \mathrm{n}$-Octyl- $\beta$-D-glucoside, $0.1 \mathrm{M}$ sodium citrate tribasic dihydrate, 22\% PEG3350, $100 \mathrm{mM} \mathrm{NaCl}$ and $15 \%$ ethylene glycol; and flash-frozen in liquid nitrogen. Diffraction data were collected by helical scanning at BL32XU (SPring-8) on an EIGER X 9M detector (Dectris) and processed with autoPROC ${ }^{51}$, STRANISO ${ }^{52}$ and the CCP4 program $^{53}$. The structure was elucidated by molecular replacement with Molrep $^{54}$ using a rabbit antibody (PDB 4ma3) as a search model, and refined by Refmac5 (ref. ${ }^{55}$ ), phenix.refine ${ }^{56}$ and $\mathrm{Coot}^{57}$. The final model for Fab14 included all amino acid residues, except for residues from positions 127 to 132 of the heavy chain.

The hXkr8-hBSG $\Delta$-Fab14 was crystallized by the sitting drop vapor diffusion method with MemGold system (Molecular Dimensions). hXkr8-hBSG $\Delta-$ Fab14 $\left(8.1 \mathrm{mg} \mathrm{ml}^{-1}\right)$ in a glass tube coated with a film of $90 \mu \mathrm{g}$ of DOPC was supplemented with $225 \mu \mathrm{g}$ of $\mathrm{C}_{12} \mathrm{E}_{8}$ (lipid detergent ratio of 1:2.5) and incubated overnight while mixing. The lipidated protein was mixed with an equal volume of $0.1 \mathrm{M}$ Tris- $\mathrm{HCl}$ ( $\mathrm{pH}$ 8.0) buffer containing $0.1 \mathrm{M}$ sodium chloride, $0.1 \mathrm{M}$ cadmium chloride hemi(pentahydrate) and $33 \%$ PEG 400 , and kept at $20^{\circ} \mathrm{C}$. Crystals were collected after 2 months and flash-frozen in liquid nitrogen. X-ray diffraction data were collected by helical scanning using KUMA and SHIKA programs at the BL32XU beamline at SPring- 8 and processed as described above. The phase was identified by molecular replacement by Molrep ${ }^{54}$ using Fab14, and domain 2 of BSG (PDB $3 \mathrm{~B} 5 \mathrm{H})$ was placed by the Coot program ${ }^{57}$. The model was refined by $\operatorname{Coot}^{57}$ and phenix.refine $e^{56}$ programs, resulting in the structure of Fab14 and the extracellular region of $h B S G \Delta$. Other parts, including $h X k r 8$ and the transmembrane region of hBSG, were not modeled because the electron density map did not visualize them. hXkr8 likely dissociated from hBSG $\Delta$ during the crystallization. The crystallographic and refinement statistics are summarized in Table 1. Molecular graphics were illustrated using PyMOL Molecular Graphics System, v.2.4.0. (Schrodinger)

Electron microscopy. In negative-staining electron microscopy, the hXkr8hBSG $\Delta$-Fab complex $\left(0.01 \mathrm{mg} \mathrm{ml}^{-1}\right)$ was applied to freshly glow-discharged, carbon-coated 200-mesh copper grids (Nisshin EM), and briefly blotted with filter paper (Whatman no. 1). Samples were stained with $2 \%$ uranyl acetate, and subjected to transmission electron microscopy (H7650, HITACHI).

For cryo-EM, immediately before preparing grids, fluorinated FC 8 was added at a final concentration of $0.075 \%$ to hXkr8-hBSG $\Delta$-Fab complex $\left(8 \mathrm{mg} \mathrm{ml}^{-1}\right)$. 
Samples were applied to the glow-discharged Quantifoil holey carbon grid $(\mathrm{Cu} / \mathrm{Rh}$, R1.2/1.3, 200-mesh), blotted by Vitrobot (Thermo Fisher Scientific) for $4 \mathrm{~s}$ under $100 \%$ humidity at $6^{\circ} \mathrm{C}$ with a blot force of +10 and plunge-frozen in liquid ethane. Movies were acquired by a Titan Krios (Thermo Fisher Scientific) equipped with the $\mathrm{K} 3$ summit direct electron detector (Gatan) operated at $300 \mathrm{kV}$ and working under low-dose conditions ( 48 frames at $48 \mathrm{e}^{-} / \AA^{2}$ ).

Movies were subjected to beam-induced motion correction using RELION 3.1, and contrast transfer function parameters were estimated by CTFFIND4 (ref. ${ }^{58}$ ). All of the following processes were performed using RELION 3.1. Particles were picked from 200 randomly selected micrographs using template-free Laplacian-of-Gaussian picking, and subjected to multiple rounds of reference-free two-dimensional (2D) classification. Good 2D classes were selected as templates and 2D template-based particle picking was performed. In total, 864,702 particles from 1,977 micrographs were auto-picked, extracted with down-sampling to a pixel size of $3.32 \AA$ per pixel and subjected to 2D classification. Among 655,655 particles subjected to 3D classification, 192,162 particles were selected, re-extracted with a pixel size of $1.245 \AA$ per pixel and subjected to 3D refinement. The 3D map and particle set yielded were subjected to per-particle defocus refinement, beam-tilt refinement, Bayesian polishing and 3D refinement. No-align focused 3D classification with a mask covering hXkr8-hBSG $\Delta$ and the Fv domain of Fab resulted in one class of map with 62,124 particles. Final 3D refinement and postprocessing yielded a map with an overall resolution of $3.8 \AA$, estimated by the gold-standard FSC $=0.143$ criterion. The processing strategy is described in Extended Data Fig. 3.

Model building and refinement. At a resolution of $3.8 \AA$, the cryo-EM density map had sufficient quality for the de novo model building of hXkr8 and the transmembrane helix of hBSG. A polyalanine helix model was initially built by $\operatorname{Coot}^{57}$, and amino acids were subsequently assigned based on the density of bulky side chains. The structure of the extracellular domain of hBSG and Fab, elucidated by the $\mathrm{X}$-ray diffraction analysis, was fit to the map by rigid body refinement (phenix.real_space_refine) ${ }^{59}$. The model was then manually fit to the map using $\operatorname{Coot}^{57}$, and simulated annealing at $500 \mathrm{~K}$ using the program of phenix.real_space refine. The first 6 amino acids and last 35 amino acids of hXkr8, and the last 35 amino acids of hBSG, were not modeled. Molecular graphics were illustrated using UCSF Chimera $^{60}$ and ChimeraX ${ }^{61}$.

Expression level of Xkr8. For the western blotting with the whole-cell lysates, cells were lysed on ice for $10 \mathrm{~min}$ in radioimmunoprecipitation assay (RIPA) buffer (50 mM HEPES-NaOH buffer ( $\mathrm{pH} 8.0$ ), $1 \%$ Nonidet P-40, $0.1 \%$ SDS, $0.5 \%$ sodium deoxycholate and $150 \mathrm{mM} \mathrm{NaCl}$ ) containing protease inhibitors, and insoluble materials were removed by centrifugation. The lysates were incubated at room temperature for $30 \mathrm{~min}$ in SDS sample buffer ( $40 \mathrm{mM}$ Tris-HCl buffer ( $\mathrm{pH}$ 6.8), $2 \%$ SDS, $5 \%$ glycerol, $1 \% 2$-mercaptoethanol and $0.01 \%$ bromophenol blue), and separated by $10-20 \%$ SDS-PAGE (Nacalai Tesque). Proteins were transferred to a PVDF membrane (Merck), blotted with 10,000-fold-diluted HRP-rabbit anti-GFP antibody (anti-GFP pAb-HRP-DirecT) and visualized using Immobilon Western Chemiluminescent HRP substrate (Merck).

For western blotting with the light membrane fractions, cells were homogenized with a Dounce homogenizer in $10 \mathrm{mM}$ Tris-HCl buffer (pH 7.5) containing $1 \mathrm{mM} p \mathrm{APMSF}$ and mixed with an equal volume of $10 \mathrm{mM}$ Tris- $\mathrm{HCl}$ buffer (pH 7.5) containing $0.5 \mathrm{M}$ sucrose, $0.1 \mathrm{M} \mathrm{KCl}, 10 \mathrm{mM} \mathrm{MgCl}, 1 \mathrm{mM}$ EGTA and $1 \mathrm{mM} p$ APMSF. After successive centrifugations at $800 \mathrm{~g}$ for $10 \mathrm{~min}$ and at $8,000 \mathrm{~g}$ for $10 \mathrm{~min}$, membrane fractions were collected by centrifugation at $100,141 \mathrm{~g}$ for $1 \mathrm{~h}$. Crude membranes were suspended in $10 \mathrm{mM}$ Tris-HCl buffer ( $\mathrm{pH} 7.5)$ containing $50 \mathrm{mM} \mathrm{KCl}, 5 \mathrm{mM} \mathrm{MgCl}_{2}, 0.5 \mathrm{mM}$ EGTA, $1 \mathrm{mM}$ pAPMSF and $40 \%$ $(\mathrm{w} / \mathrm{v})$ sucrose, and were loaded between $17 \%$ and $50 \%(\mathrm{w} / \mathrm{v})$ sucrose layers in centrifugation buffer. After centrifugation at $100,141 \mathrm{~g}$ at $4^{\circ} \mathrm{C}$ for $150 \mathrm{~min}$, the light membrane fraction at the $17 \% / 40 \%(\mathrm{w} / \mathrm{v})$ sucrose interface was collected, diluted with centrifugation buffer and collected by centrifugation. The precipitates were homogenized in TBS containing $10 \%$ glycerol and protease inhibitors by passing through a non-dead-space $29 \mathrm{G}$ needle (TERUMO). A $1 / 10$ volume of $10 \%$ LMNG $-1 \% \mathrm{CHS}$ was added to the sample and rotated at $4{ }^{\circ} \mathrm{C}$ for $2 \mathrm{~h}$. After removing insoluble materials by centrifugation, the supernatants were recovered as light membrane lysates.

Crosslinking experiment. Crosslinking of Cys-substituted hXkr8 and hBSG was performed as described ${ }^{62}$. Briefly, HEK293T cells were transfected with the expression vectors for hXkr8 and hBSG, cultured for $2 \mathrm{~d}$, suspended in TBS with protease inhibitors and disrupted by sonication for $30 \mathrm{~s}$ at an amplitude of 20 using an ultrasonic disrupter (Q55; Qsonica). After centrifugation at 6,000 $\mathrm{g}$ for $25 \mathrm{~min}$, the crude membrane fractions were collected by centrifugation at $99,731 \mathrm{~g}$ for $1 \mathrm{~h}$; homogenized in TBS-10\% glycerol, $1 \mathrm{mM}$ EGTA and protease inhibitors; and treated at room temperature for $5 \mathrm{~min}$ with $1 \mathrm{mM}$ reduced glutathione. The membranes were incubated at room temperature for $10 \mathrm{~min}$ with copper phenanthroline $(0.3 \mathrm{mM}$ $\mathrm{CuSO}_{4}$ and $0.9 \mathrm{mM} 1,10$-phenanthroline). After adding 0.25 vol. of $200 \mathrm{mM}$ Tris-HCl buffer ( $\mathrm{pH}$ 6.8) containing $10 \% \mathrm{SDS}, 25 \%$ glycerol, $25 \mathrm{mM}$ N-ethylmaleimide and $0.05 \%$ bromophenol blue, the lysates $(0.75 \mu \mathrm{g})$ were separated by $10-20 \%$ SDS-PAGE, transferred to a PVDF membrane, blotted with 10,000-fold-diluted
HRP-rabbit anti-GFP antibody or 3,000-fold-diluted HRP-mouse anti-HA monoclonal antibody (Direct-Blot HRP anti-HA.11 Epitope Tag antibody) and visualized by Immobilon Western Chemiluminescent HRP substrate.

Establishment of stable transformants and the phospholipid scrambling assay. PLB and $B S G^{-/-} N P T N^{-/-}$W3 cells were transformed with a pantropic retrovirus as described ${ }^{9}$. Briefly, HEK293T cells were transfected with a pMXs-Puro or pCX4-bsr vector together with pGP (Takara Bio), and pCMV-VSV-G-RSV-Rev (RIKEN). Virus was concentrated by centrifugation at $6,000 \mathrm{~g}$ for $16 \mathrm{~h}$, and used to infect cells. Stable transformants were selected with $1 \mu \mathrm{g} \mathrm{ml}^{-1}$ puromycin or $10 \mu \mathrm{g} \mathrm{ml}^{-1}$ blasticidin. TMEM16F $\mathrm{F}^{-/-} \mathrm{Xkr}^{-/-} \mathrm{Ba} / \mathrm{F} 3$ cells $^{16}$ were transfected by electroporation using NEPA21 (Nepa Gene), and selected with $0.5-1 \mathrm{mg} \mathrm{ml}^{-1} \mathrm{G} 418$ or $1.0 \mathrm{\mu g} \mathrm{ml}^{-1}$ puromycin. Stable transformants were observed to localize Xkr8-GFP by confocal microscopy (FV-1000D; Olympus).

Apoptosis was induced by incubating cells with 10 units per ml of FasL for $1 \mathrm{~h}$ at $37^{\circ} \mathrm{C}$ as described ${ }^{9}$. Phospholipid scrambling was assayed by the exposure of PtdSer or PtdEtn, or the internalization of NBD-SM ${ }^{9,41,63}$. The PtdSer exposure was detected by incubating cells with 1,000-fold-diluted Cy5-Annexin V in Annexin $\mathrm{V}$ buffer (10 mM HEPES-NaOH buffer (pH 7.4), $140 \mathrm{mM} \mathrm{NaCl}$ and $2.5 \mathrm{mM}$ $\mathrm{CaCl}_{2}$ ) containing $5 \mu \mathrm{g} \mathrm{ml}^{-1}$ propidium iodide followed by flow cytometry using FACSCanto II (BD Biosciences) ${ }^{9,41}$. The data were analyzed by FlowJo v.7.6.5 and FACSDiva v.6.1.3 (BD Biosciences). For NBD-SM-incorporation, cells $\left(1.0 \times 10^{6}\right.$ cells per ml) were incubated with $0.5 \mu \mathrm{M}$ NBD-SM in Annexin V buffer. After incubation, an aliquot was mixed with an equal volume of Annexin $\mathrm{V}$ buffer containing $5 \mathrm{mg} \mathrm{m}^{-1}$ fatty-acid-free BSA (Sigma-Aldrich) and $5 \mathrm{nM}$ SYTOX red (Thermo Fisher Scientific), and analyzed by FACSCanto II (ref. ${ }^{9}$ ). The gating strategies in flow cytometry for the cells that bound Annexin V or incorporated NBD-SM are presented in Supplementary Fig. 4 . The PtdEtn exposure was examined by Ro $09-0198$-induced cytotoxicity as described ${ }^{36}$. In brief, cells at $1.0 \times 10^{5}$ per $\mathrm{ml}$ in Annexin $\mathrm{V}$ buffer containing $5 \mathrm{mg} \mathrm{ml}^{-1} \mathrm{BSA}$ were incubated with Ro 09-0198-biotin, and the lactate dehydrogenase (LDH) activity in the supernatant was measured with the LDH Cytotoxicity Detection Kit (Takara). The respective scrambling activity of mutant mXkr8-GFP was normalized by its expression level (mean fluorescence intensity of their GFP) compared with that of the wild-type mXkr8-GFP except for parental $\mathrm{Ba} / \mathrm{F} 3$ cells.

LC-MS/MS analysis of phospholipids. To extract lipids from hXkr8-hBSG $\Delta$, the protein was mixed and vortexed with $1 \mathrm{ml}$ of methanol/chloroform/water $(10: 5: 3, \mathrm{v} / \mathrm{v} / \mathrm{v})$ as described ${ }^{64,65}$. The mixture was sonicated at $4{ }^{\circ} \mathrm{C}$ with a Bioruptor II (Cosmo Bio) at maximum power for $30 \mathrm{~s}$ five times with 30 -s intervals, and spun at $16,000 \mathrm{~g}$ for $5 \mathrm{~min}$. To a $700-\mu \mathrm{l}$ aliquot of the supernatant, $195 \mu \mathrm{l}$ of chloroform and $195 \mu \mathrm{l}$ of water were added, and the aqueous and organic layers were separated by centrifugation. The $200 \mu \mathrm{l}$ of the organic layer was evaporated in a SpeedVac concentrator and dissolved in $100 \mu \mathrm{l}$ of $10 \mathrm{mM}$ ammonium formate containing $25 \%(\mathrm{v} / \mathrm{v})$ isopropanol and $25 \%(\mathrm{v} / \mathrm{v})$ acetonitrile. LC-MS/MS analysis was performed using an UltiMate 3000 HPLC coupled with a Q Exactive Hybrid Quadrupole Orbitrap mass spectrometer (Thermo Fisher Scientific). The mass spectrometer was equipped with an electrospray ionization source. The LCMS conditions with Phenomenex Kinetex C8 column (Shimadzu GLC) were: injection volume, 1 or $2 \mu \mathrm{l}$; mobile phase A, $20 \mathrm{mM}$ ammonium formate; mobile phase B, isopropanol/acetonitrile (1:1, v/v); flow rate, $0.2 \mathrm{ml} \mathrm{min}^{-1}$; modifier (B) gradient: $20 \%$ at $0-1 \mathrm{~min}, 45 \%$ at $2 \mathrm{~min}, 92.5 \%$ at $25 \mathrm{~min}, 100 \%$ at $26-35 \mathrm{~min}$, $20 \%$ at $35.1-41 \mathrm{~min}$; column temperature, $45^{\circ} \mathrm{C}$. The MS tune conditions were: ionization mode, positive and negative; capillary voltage, $3.5 \mathrm{kV}$ for both positive and negative ionization; capillary temperature, $350^{\circ} \mathrm{C}$. The following full mass scan/data-dependent MS/MS (full MS/dd-MS ${ }^{2}$ ) mode was used for phospholipid analysis: scan range, 500-1,100 m/z; mass resolution, 70,000; AGC target, $3 \times 10^{6}$; maximum injection time, $100 \mathrm{~ms}$. The parameters for data-dependent product ion scanning were: mass resolution, 17,500; AGC target, $1 \times 10^{5}$; maximum injection time, $100 \mathrm{~ms}$; loop count, 5 ; isolation width, $2.0 \mathrm{~m} / z$; stepped normalized collision energy, 15, 25 and 35. The LC-MS/MS system was controlled by Chromeleon 6.8 and Xcalibur 4.0 software (Thermo Fisher Scientific). Phospholipid species were analyzed by Xcalibur Qual Browser 4.3 software.

Statistics and reproducibility. Statistical analysis was performed using RStudio v.1.1.463. Error bars indicate standard deviation. For all experiments presented as representative images in Figs. 1a,b, 2e, 3b and 5g and Extended Data Fig. 1b, three to five biological replicates were performed. The experiments presented in Figs. $2 \mathrm{c}$ and $3 \mathrm{c}$ were performed twice. The experiments presented in Fig. 1a (SDS-PAGE) and Extended Data Fig. 1d,e were performed more than five times. All other experiments were performed at least three times as described in the legend for each figure.

Reporting Summary. Further information on research design is available in the Nature Research Reporting Summary linked to this article.

\section{Data availability}

The cryo-EM density map for hXkr8-hBSG $\Delta$-Fab18 was deposited in the Electron Microscopy Data Bank (accession number: EMD-30636). The coordinates for the 
models of the hXkr8-hBSG $\Delta$-Fab18 complex were deposited in the Protein Data Bank (PDB) under the accession code PDB 7DCE. The coordinates and structural factors of Fab14 and the hBSG (domain2)-Fab14 complex were deposited in PDB under accession codes PDB 7D9Z and PDB 7DAA, respectively. The mass spectrometry data were deposited in the ProteomeXchange Consortium under accession code PXD 027776. Source data are provided with this paper. Several structural coordinates in the PDB database were used in this study, which can be located by accession numbers $3 \mathrm{~B} 5 \mathrm{H}, 4 \mathrm{ma} 3$ and $1 \ln 2$.

\section{References}

48. Sawitri, W. D. et al. Purification and characterization of recombinant sugarcane sucrose phosphate synthase expressed in E. coli and insect Sf9 cells: an importance of the $\mathrm{N}$-terminal domain for an allosteric regulatory property. J. Biochem. 159, 599-607 (2016).

49. Murai, K., Murakami, H. \& Nagata, S. Myeloid-specific transcriptional activation by murine myeloid zinc finger protein-2. Proc. Natl Acad. Sci. USA 95, 3461-3466 (1998).

50. Seeber, S. et al. A robust high throughput platform to generate functional recombinant monoclonal antibodies using rabbit $\mathrm{B}$ cells from peripheral blood. PLoS ONE 9, e86184-86114 (2014).

51. Vonrhein, C. et al. Data processing and analysis with the autoPROC toolbox. Acta Crystallogr. D Biol. Crystallogr. D67, 293-302 (2011).

52. Tickle, I. J. et al. STARANISO (Global Phasing Ltd, 2018); http://staraniso. globalphasing.org/cgi-bin/staraniso.cgi

53. Collaborative Computational Project, Number 4. The CCP4 suite: programs for protein crystallography. Acta Crystallogr. D Biol. Crystallogr. D50, 760-763 (1994).

54. Vagin, A. \& Teplyakov, A. Molecular replacement with MOLREP. Acta Crystllogr. D Biol. Crystallogr. D66, 22-25 (2009).

55. Murshudov, G. N., Vagin, A. A. \& Dodson, E. J. Refinement of macromolecular structures by the maximum-likelihood method. Acta Crystallogr. D Biol. Crystallogr. D53, 240-255 (1997).

56. Afonine, P. V. et al. Towards automated crystallographic structure refinement with phenix.refine. Acta Crystallogr. D Biol. Crystallogr. D68, 352-367 (2012).

57. Emsley, P., Lohkamp, B., Scott, W. G. \& Cowtan, K. Features and development of Coot. Acta Crystallogr. D Biol. Crystallogr. D66, 486-501 (2010).

58. Rohou, A. \& Grigorieff, N. CTFFIND4: fast and accurate defocus estimation from electron micrographs. J. Struct. Biol. 192, 216-221 (2015).

59. Afonine, P. V. et al. Real-space refinement in PHENIX for cryo-EM and crystallography. Acta Crystallogr. D Struct. Biol. D74, 531-544 (2018).

60. Pettersen, E. F. et al. UCSF Chimera-a visualization system for exploratory research and analysis. J. Comput. Chem. 25, 1605-1612 (2004).

61. Goddard, T. D. et al. UCSF ChimeraX: meeting modern challenges in visualization and analysis. Protein Sci. 27, 14-25 (2017).

62. Toyoshima, C. et al. Crystal structures of the calcium pump and sarcolipin in the $\mathrm{Mg}^{2+}$-bound E1 state. Nature 495, 260-264 (2013).

63. Emoto, K., Toyama-Sorimachi, N., Karasuyama, H., Inoue, K. \& Umeda, M. Exposure of phosphatidylethanolamine on the surface of apoptotic cells. Exp. Cell. Res. 232, 430-434 (1997).

64. Bligh, E. G. \& Dyer, W. J. A rapid method of total lipid extraction and purification. Can. J. Biochem. Physiol. 37, 911-917 (1959).
65. Takeda, H. et al. Widely-targeted quantitative lipidomics method by supercritical fluid chromatography triple quadrupole mass spectrometry. $J$. Lipid Res. 59, 1283-1293 (2020).

\section{Acknowledgements}

We thank C. Gerle (Institute for Protein Research, Osaka University) for his advice on EM negative staining and the GraDeR procedure; J. Suzuki (Kyoto University) for characterizing mouse BSG mutants on N-glycosylation sites; K. Abe (Nagoya University) for his advice on the crystallization procedure; the beamline scientists at BL32XU (SPring-8)(proposals 2017A2510, 2017B2510, 2019B2538), particularly K. Hirata, for their technical assistance with data collection; K. Nagao and M. Umeda (Graduate School of Engineering, Kyoto University) for biotin-Ro09-0198; F. Takenaga and S. Tsukita (Graduate School of Frontier Biosciences, Osaka University) for their advice on EM negative staining; Y. Sakamaki, H. Kawauchi and K. Yamada for their technical assistance; and M. Kamada and M. Fujii for their secretarial assistance. This work was supported in part by Grants-in-Aid from the Japan Society for the Promotion of Science (grant no. 20K15731 to T.S.; grant nos. 15H05785 and 21F04770 to S.N), Grants-in-Aid from Core Research for Evolutional Science and Technology, the Japan Science and Technology Agency (grant no. JPMJCR14M4 to S.N.; grant no. JPMJCR14M1 to M.K.) and the Platform Project for Supporting Drug Discovery and Life Science Research (Basis for Supporting Innovative Drug Discovery and Life Science Research (BINDS)) of AMED (grant no. JP20am0101072; support number 0586 to S.N.). This work was also performed in part under the Collaborative Research Program of the Institute for Protein Research, Osaka University (no. CR-18-05), and supported in part by the Joint Usage and Joint Research Programs of the Institute of Advanced Medical Sciences, Tokushima University, to S.N.

\section{Author contributions}

T.S. designed and performed most of the experiments and wrote the manuscript. E.O. helped with protein purification. H.N. and A.N. supervised the X-ray crystal analysis and data processing. A.T. and M.K. performed and supervised the cryo-EM analysis. R.K. performed and supervised the structure modeling. C.T. supervised the whole structural analysis. T.M. and T.B. prepared Fab against hBSG. K.N. and H.K. performed LC-MS/ MS analysis. S.N. conceived and designed the project and wrote the manuscript.

\section{Competing interests}

The authors declare no competing interests.

\section{Additional information}

Extended data is available for this paper at https://doi.org/10.1038/s41594-021-00665-8. Supplementary information The online version contains supplementary material available at https://doi.org/10.1038/s41594-021-00665-8.

Correspondence and requests for materials should be addressed to Shigekazu Nagata.

Peer review information Nature Structural \& Molecular Biology thanks the anonymous reviewers for their contribution to the peer review of this work. Peer reviewer reports are available. Florian Ullrich was the primary editor on this article and managed its editorial process and peer review in collaboration with the rest of the editorial team.

Reprints and permissions information is available at www.nature.com/reprints. 


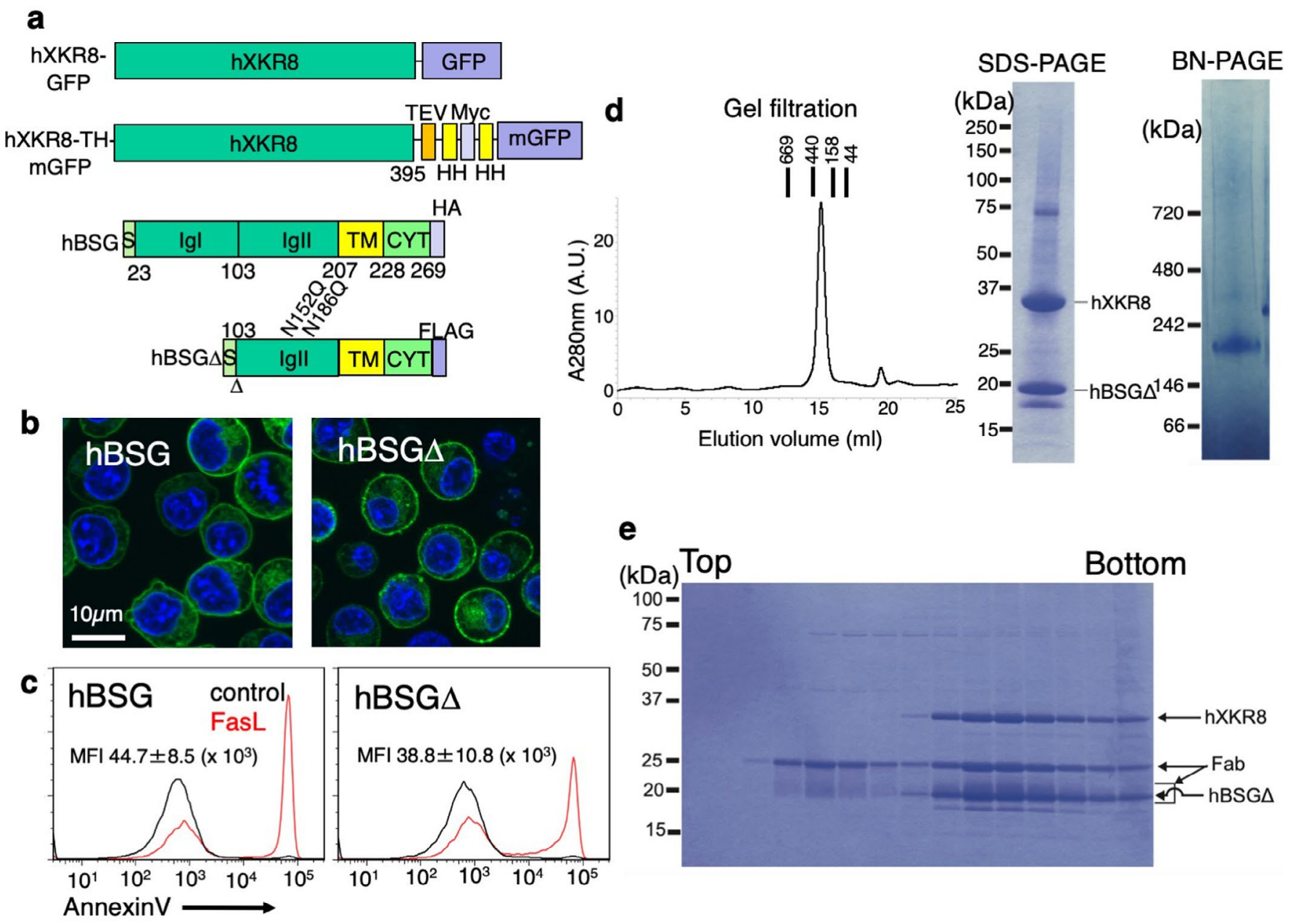

Extended Data Fig. 1 | Purification of the hXkr8-hBSG $\Delta$-Fab complex. a, Structures of hXkr8-GFP, hXkr8-TH-mGFP, hBSG, and hBSG $\Delta$. mGFP, monomeric EGFP; TEV, TEV cleavage site; HH, Histidine tag (8 His); Myc, Myc-tag; S, Signal sequence; Ig, Immunoglobulin (Ig) domain; TM transmembrane region; $\mathrm{CYT}$, cytoplasmic region. In hBSG $\Delta$, the first Ig domain ( $|g|$ ) was deleted, and two $\mathrm{N}$-glycosylation sites (Asn) were mutated to glutamine. $\mathbf{b}, \mathbf{c}, B \mathrm{BSG}^{-/}$ NPTN $\%$ W3 cells were transformed by hXkr8-GFP with hBSG or hBSG $\Delta$ and observed by confocal microscopy (b). The transformants were treated with FasL, and the Annexin V-staining profile in the PI-negative population was analyzed by flow cytometry (c). d, The purified hXkr8-hBSG complex was analyzed by gel filtration ( $7.5 \mu \mathrm{g}$ protein), SDS-PAGE $(6 \mu \mathrm{g})$, or BN-PAGE ( $3 \mu \mathrm{g})$. The molecular weight of standard proteins is shown in kDa. e, Purified hXkr8-hBSG $\Delta$ was incubated with Fab18 and subjected to GraDeR ${ }^{29}$. An aliquot of each fraction was analyzed by SDS-PAGE. hXkr8, hBSG $\Delta$, and Fab (heavy and light chains) are indicated by arrows. Source data for $\mathbf{b}, \mathbf{d}$, and $\mathbf{e}$ are available online. 
a

$\square$ BSG Igll

$\square$ Heavy chain

$\square$ Light chain

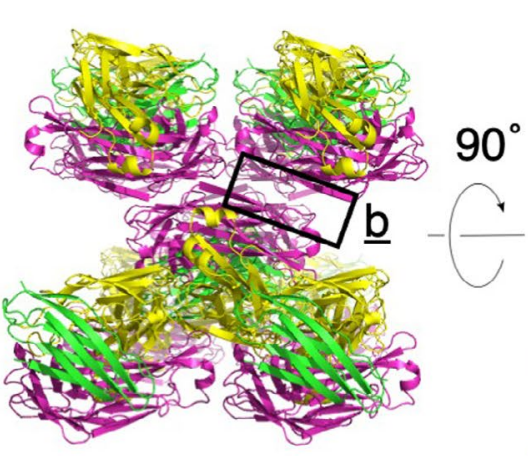

b

C
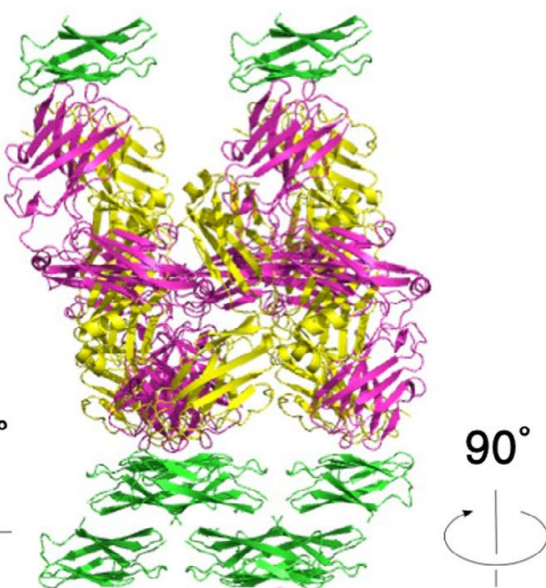

$90^{\circ}$

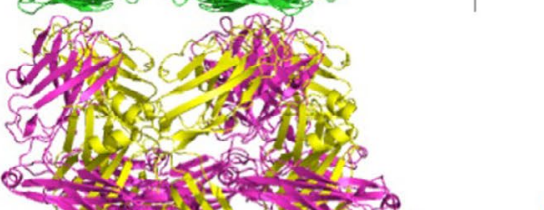

b

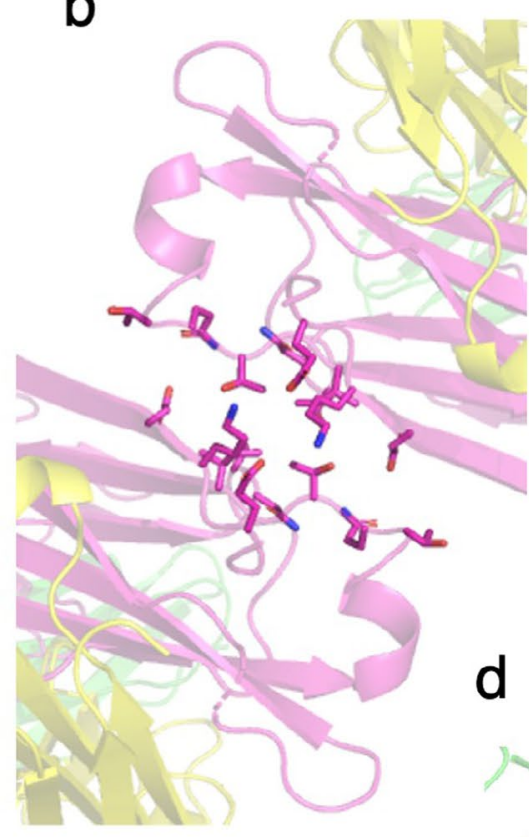

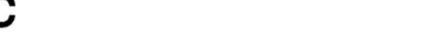

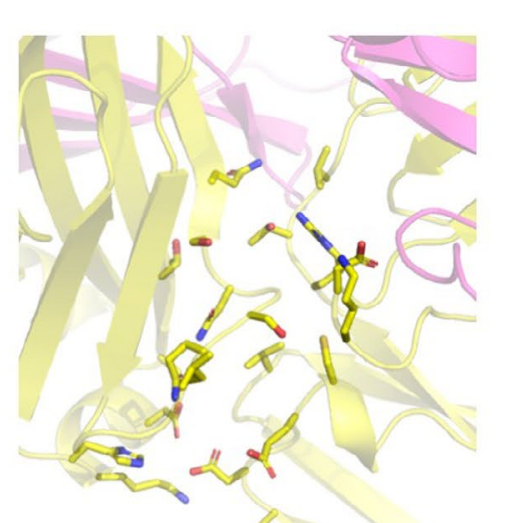

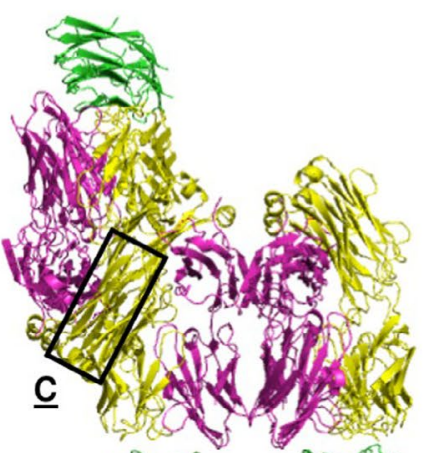
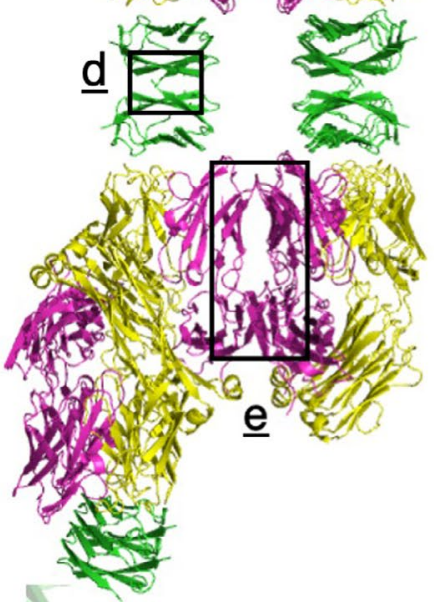

e

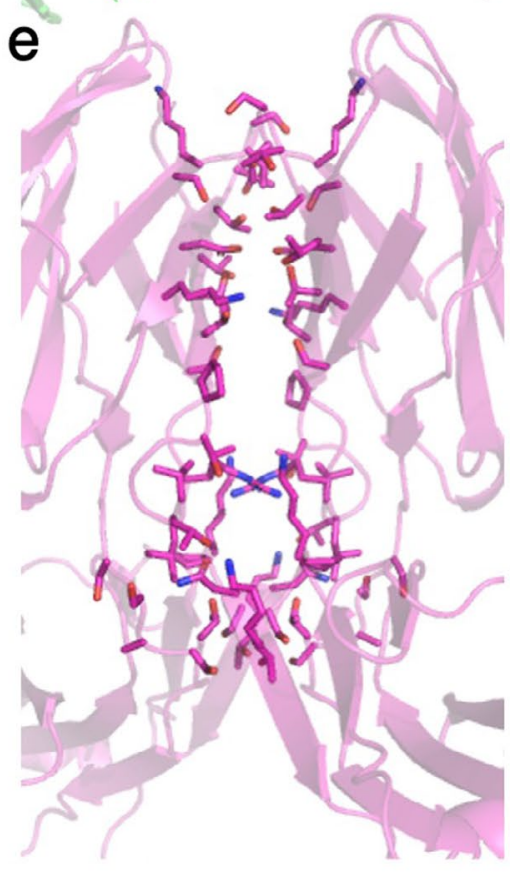

Extended Data Fig. 2 | Crystal lattice contact of the Fab14-BSG IgIl complex. a, Three views of Fab14-BSG Ig|l crystal packing. BSG IgII, the heavy chain, and light chain are shown in green, magenta, and yellow, respectively. b-e, Close-up views of the area boxed in $\mathbf{a}$. Residues at the interface of the domains are represented by sticks. 
a

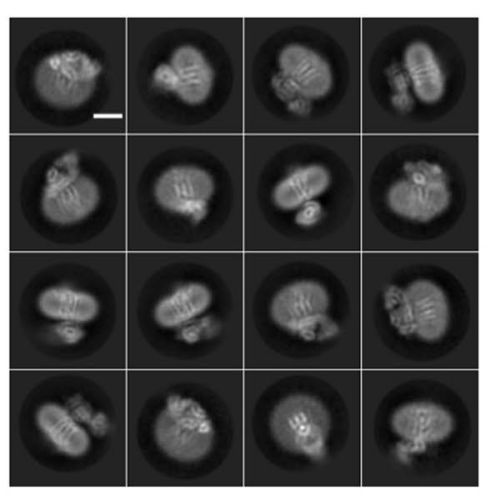

b

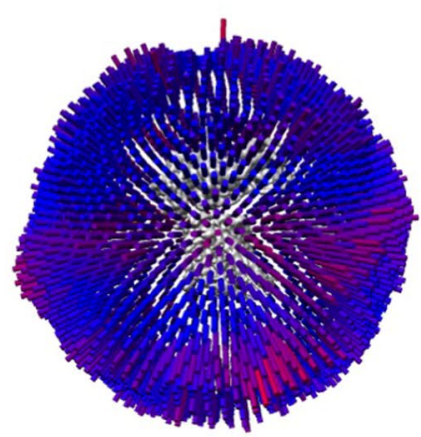

d

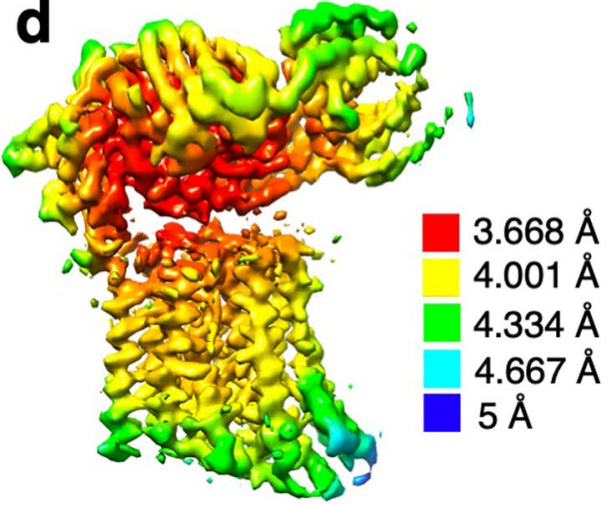

C 1998 movies Motion correction CTF estimation Particle picking
864,702 particles
2D classification

655,655 particles

3D classification
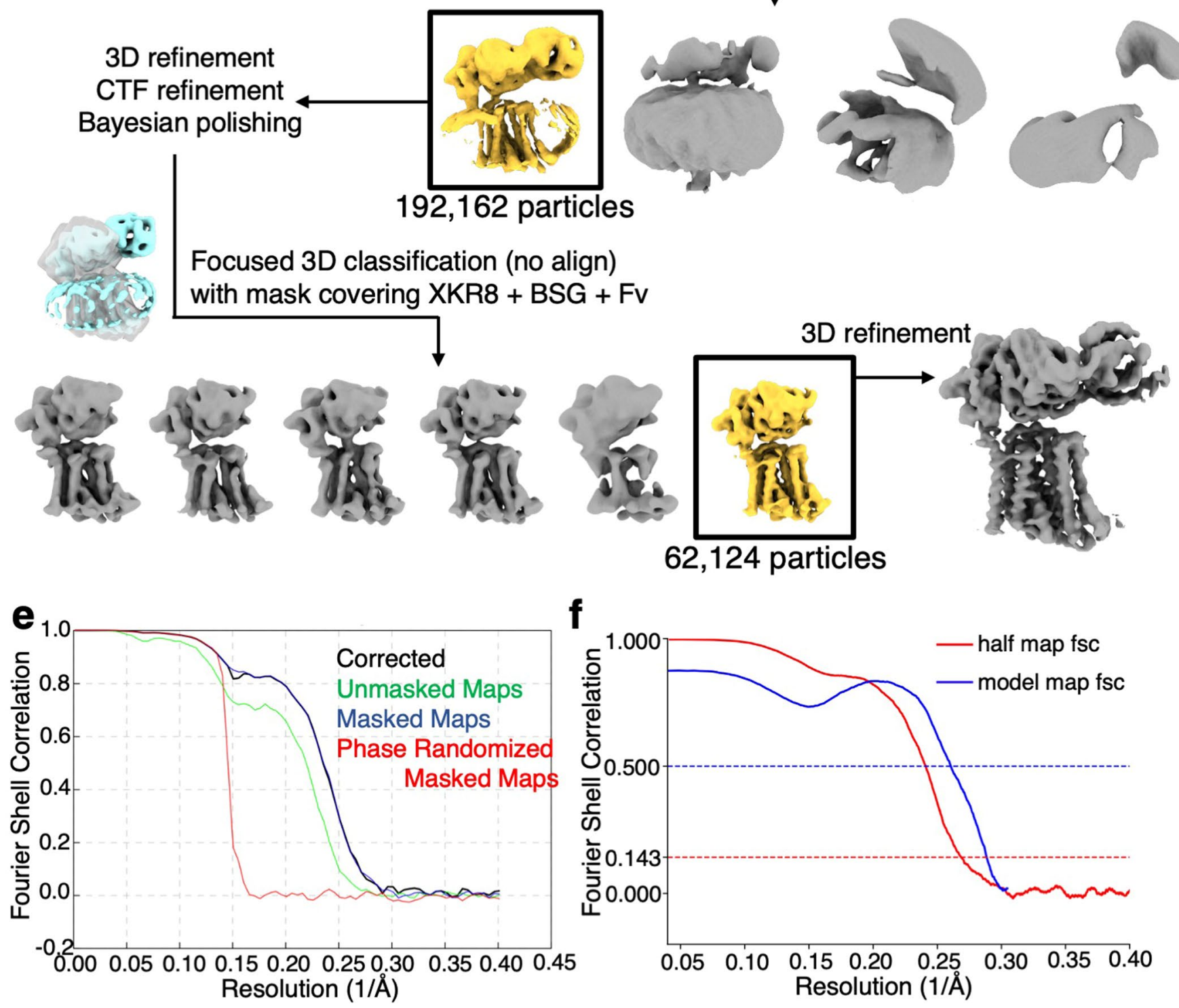

Focused 3D classification (no align) with mask covering XKR8 + BSG + Fv
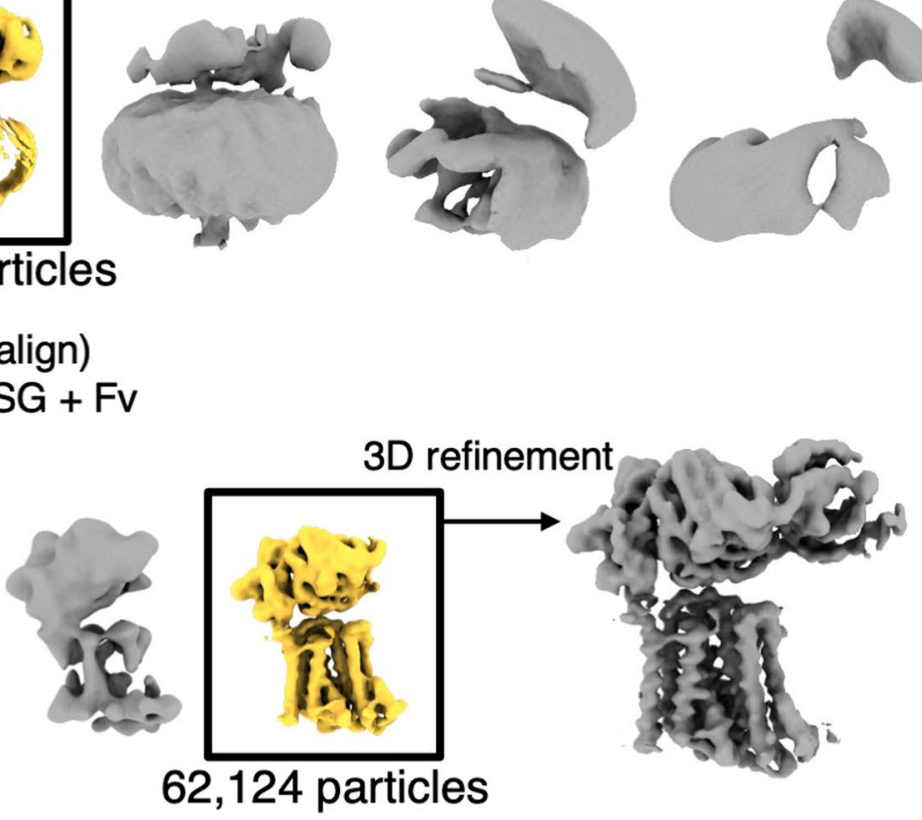

f

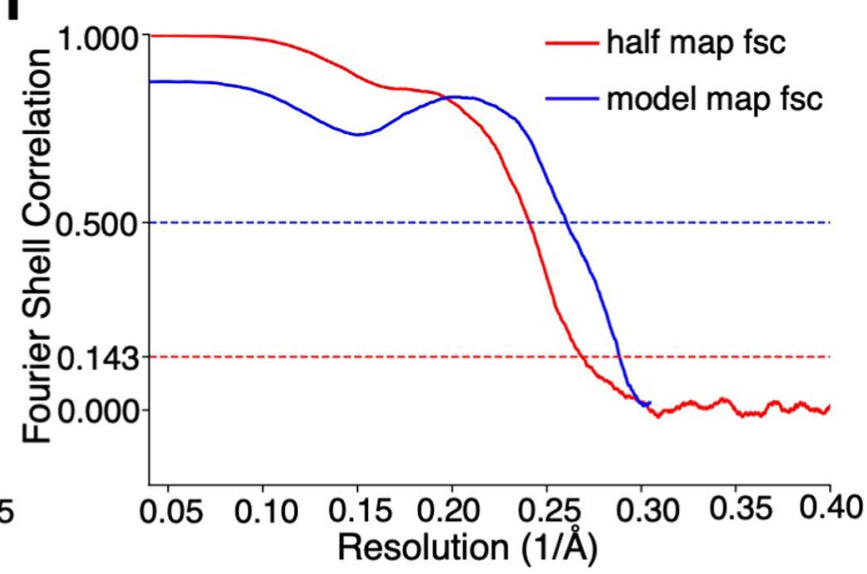

Extended Data Fig. 3 | See next page for caption. 
Extended Data Fig. 3 | Cryo-EM characterization of the hXkr8-hBSG $\Delta$-Fab complex. a, Representative 2D-class averages, bar, $5 \mathrm{~nm}$. b, Angular distribution plot of particles included in the final 3D reconstitution. The length and color of cylinders indicate the number of views at each angular orientation. Red indicates more views. c, Data processing of the hXkr8-hBSG $\Delta$-Fab complex. See Methods for the detailed procedure. d, Final reconstruction map colored by local resolution as calculated by RELION3.1. e, The Fourier shell correlation (FSC) plot between corrected (black), unmasked (green), masked (blue), and phase randomized (red) half-maps are shown. f, FSC plot for resolution estimations and model validation. The FSC curve between the EM half maps (red) and the FSC curve between the EM full map and atomic model (blue) are shown. 


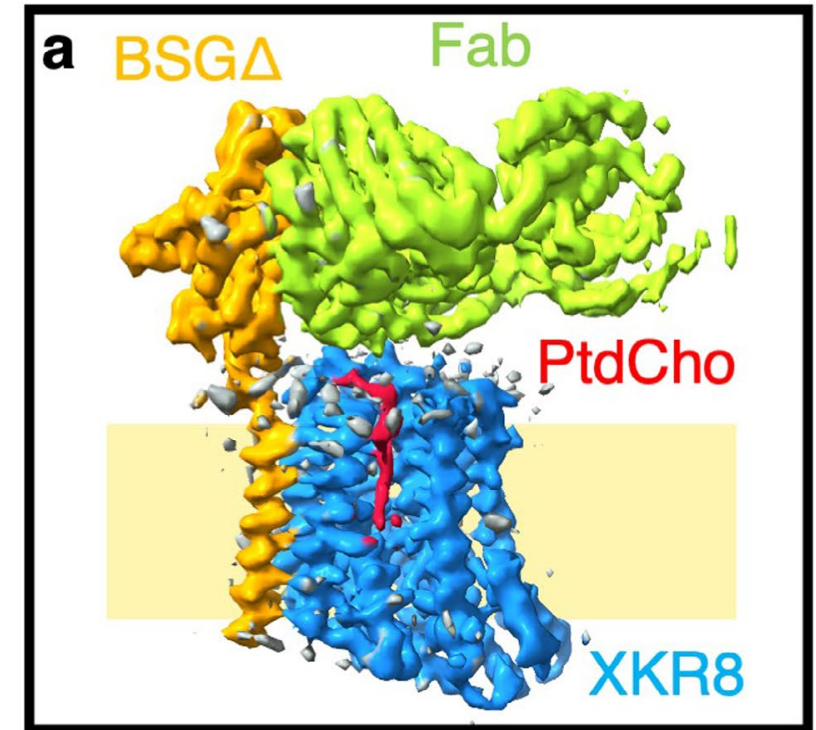

b a1 (7-39) a2 (44-69)
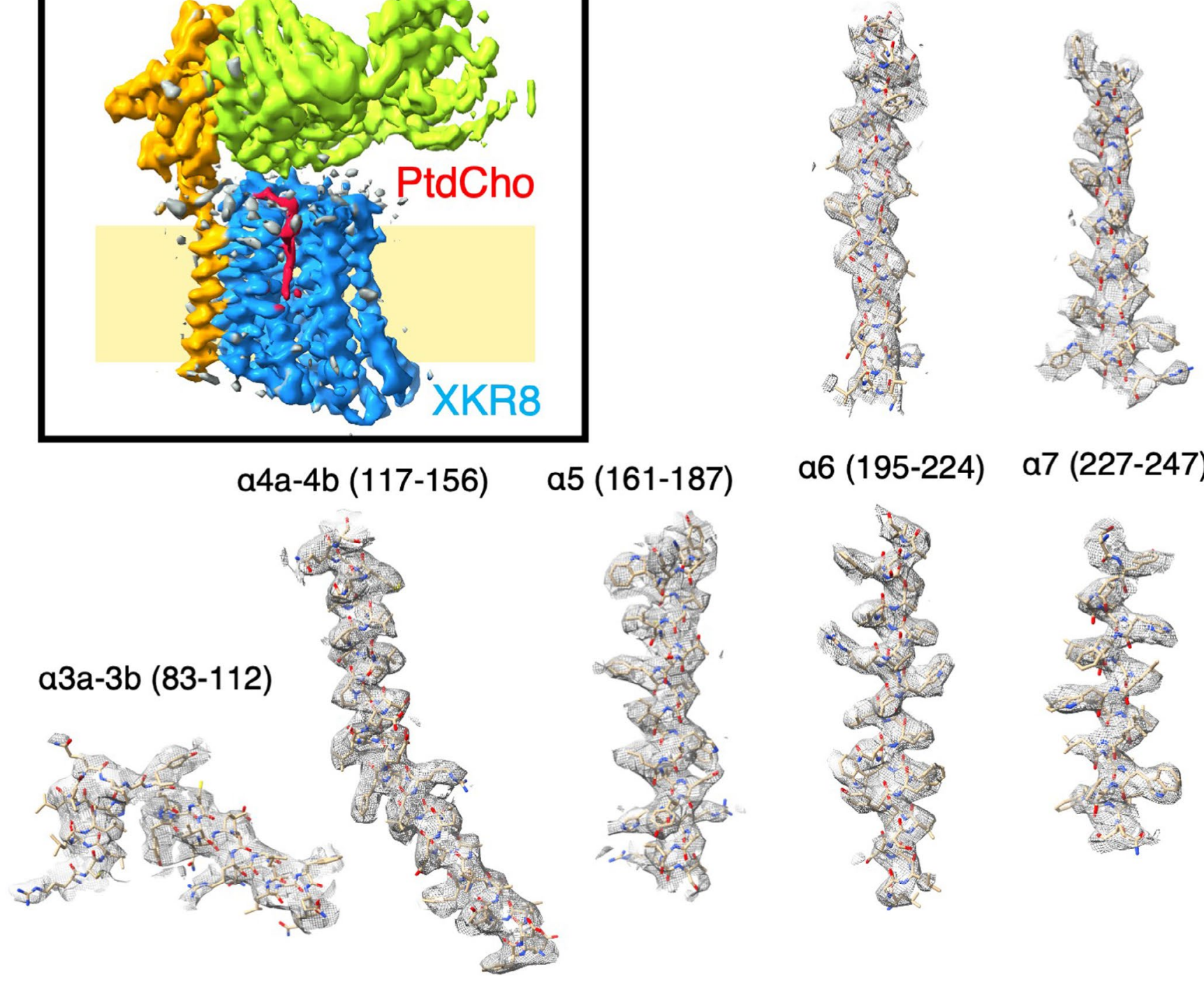

a6 (195-224) a7 (227-247)
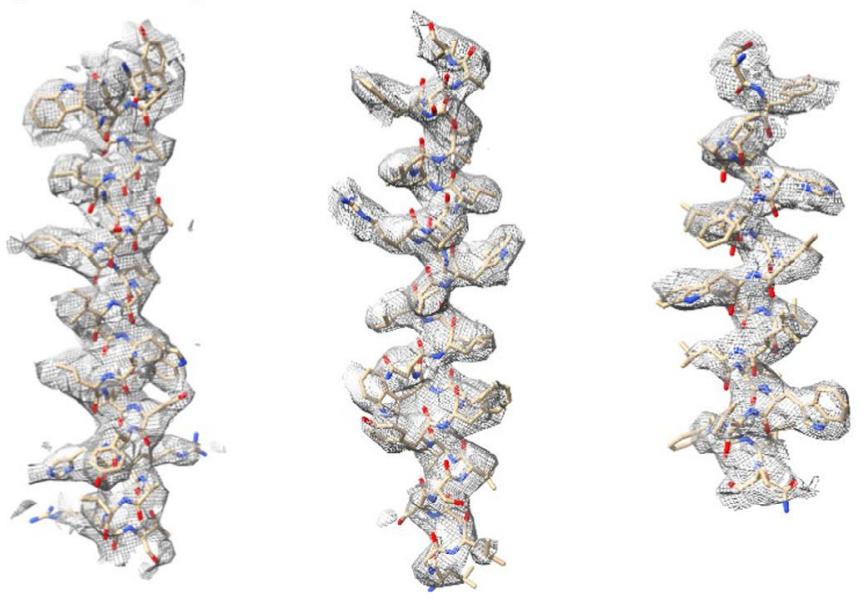

a8 (258-268) a9 (283-306) a10 (322-340)

a11 (348-358) BSG $\Delta(205-229)$
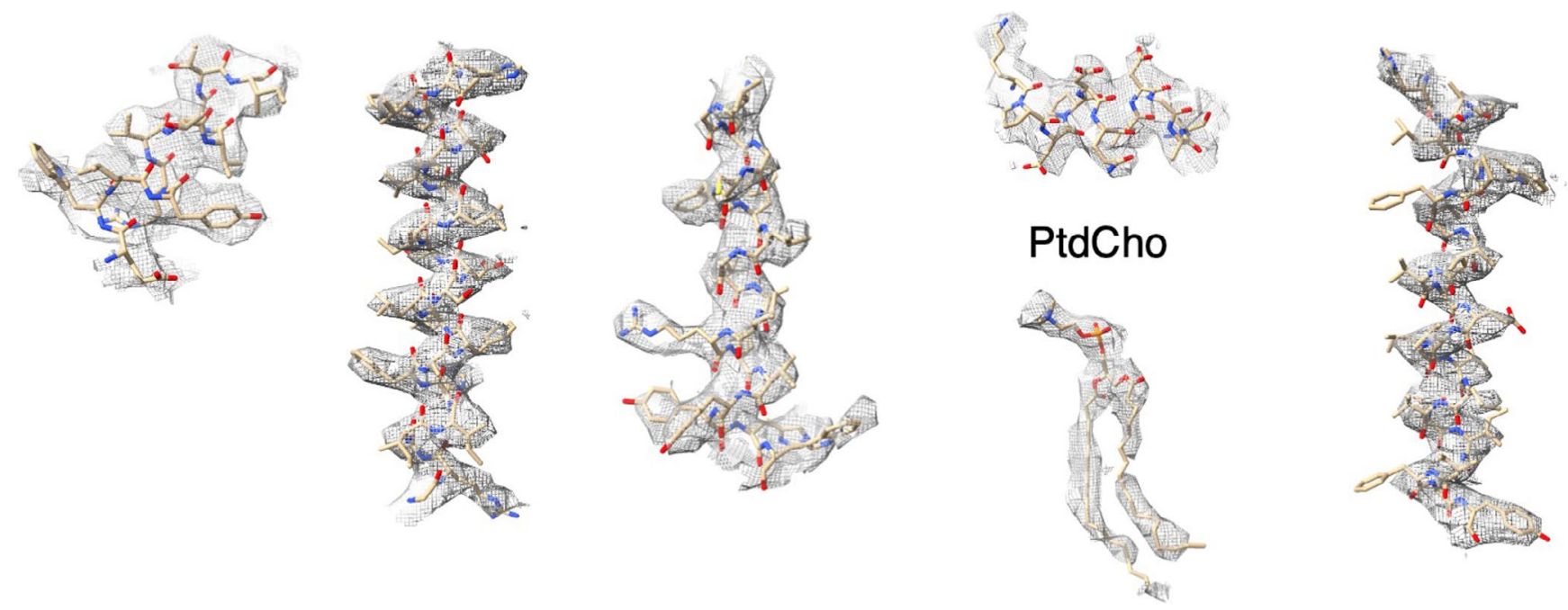

Extended Data Fig. 4 | See next page for caption. 
Extended Data Fig. 4 | Cryo-EM density of the hXkr8-hBSG $\Delta$-Fab complex. A cryo-EM map of the hXkr8-hBSG $\Delta$-Fab complex. The location of the membrane is estimated from the surface hydrophobicity and position of tryptophan residues (Extended Data Fig. 6). b, Representative cryo-EM densities of 11 helices $(\alpha 1-\alpha 11)$ of $\mathrm{hXkr} 8$, the transmembrane helix of hBSG $\Delta$, and PtdCho are superimposed on the corresponding atomic model. Electron microscopy densities are shown in grey meshes, while the model is shown as sticks colored according to the atom type: $\mathrm{C}$, $\tan ; \mathrm{N}$, blue; $\mathrm{O}$, red; and $\mathrm{S}$, yellow. 
a

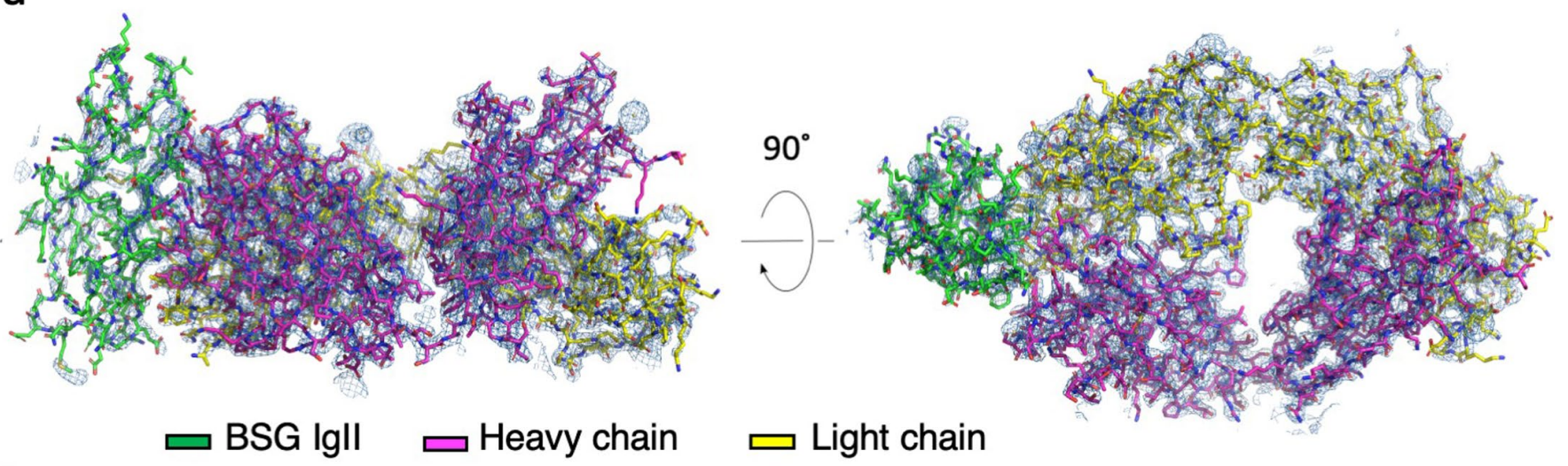

b

\section{Constant Heavy chain Variable Heavy chain} (CH1)

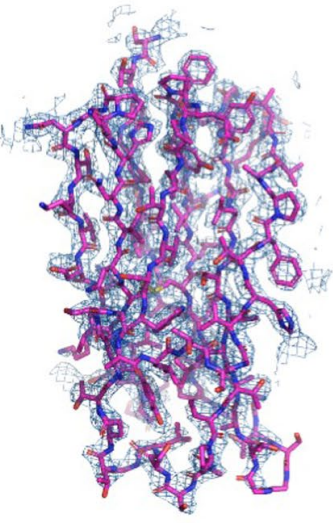

BSG Igll

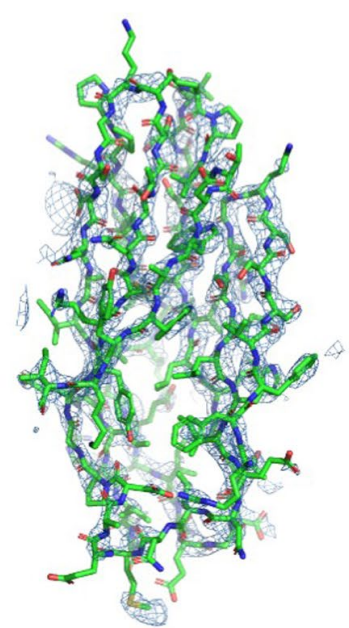

(VH)

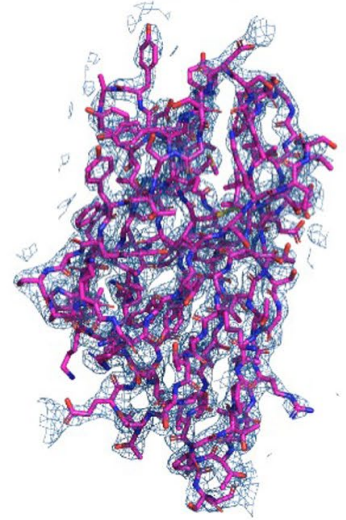

C
Variable Light chain

(VL)

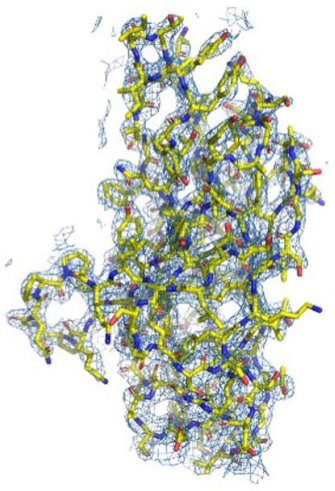

Constant Light chain

$(\mathrm{CL})$

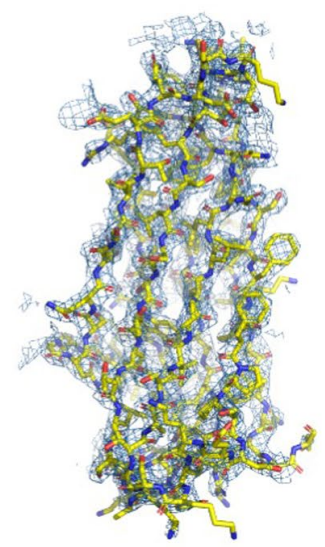
Fab-BSG interface (stereo)

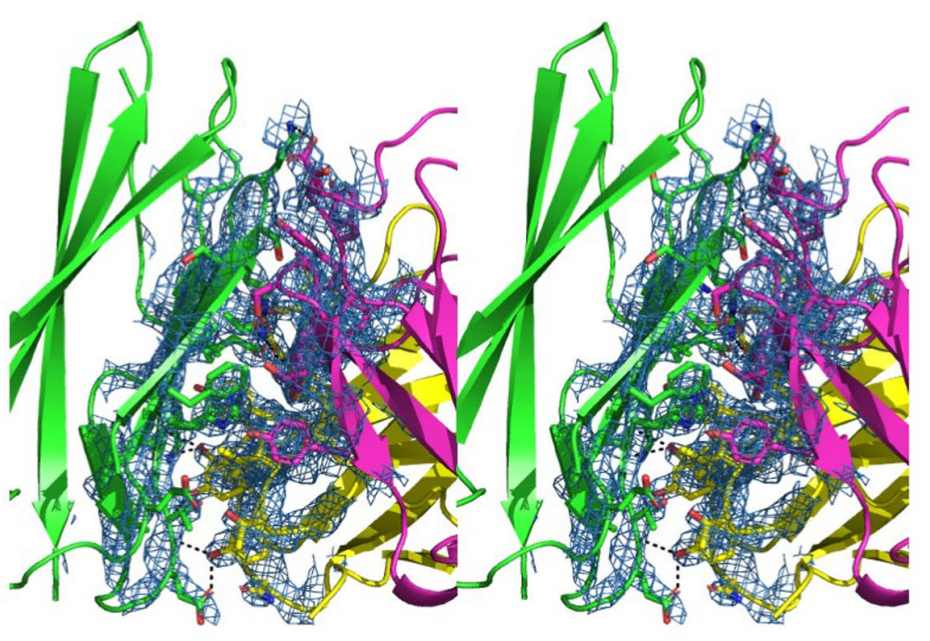

Extended Data Fig. 5 | Electron density maps for the Fab14-BSG Igll complex. a, 2Fo-Fc electron density maps of Fab14 and BSG IgIl with a 1.5 $\sigma$ contour level. BSG IgIl, the heavy chain of Fab14, and the light chain of Fab14 are shown in green, magenta, and yellow, respectively. b, 2Fo-Fc electron density maps of the indicated domains with a $1.5 \sigma$ contour level. Color code as above. c, A stereo view of the interface between Fab14 and BSG Igll. The blue mesh illustrates 2Fo-Fc electron density maps contoured at $1.5 \sigma$. Hydrogen bonds are shown as black dotted lines. Color code as above. 


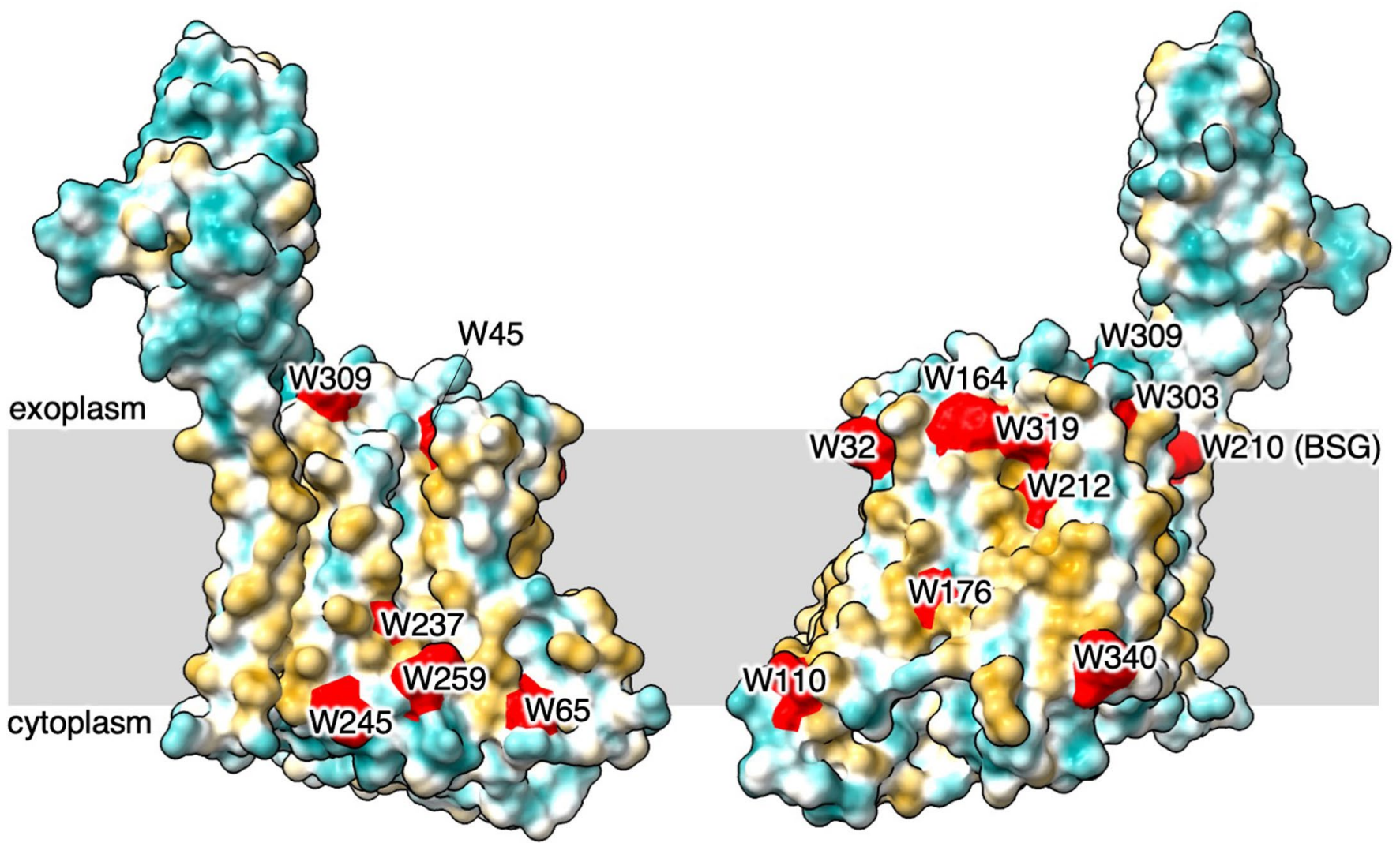

Extended Data Fig. 6 | Surface hydrophobicity and tryptophan residues in the hXkr8-basigin complex. Features of the surface hydrophobicity of the hXkr8-basigin complex represented using the UCSF Chimera X program are shown in two views. Hydrophobic residues are in brown, polar residues in blue and neutral residues in white. Tryptophan residues in $\mathrm{hXkr8}$ and the transmembrane region of basigin are shown in red. 

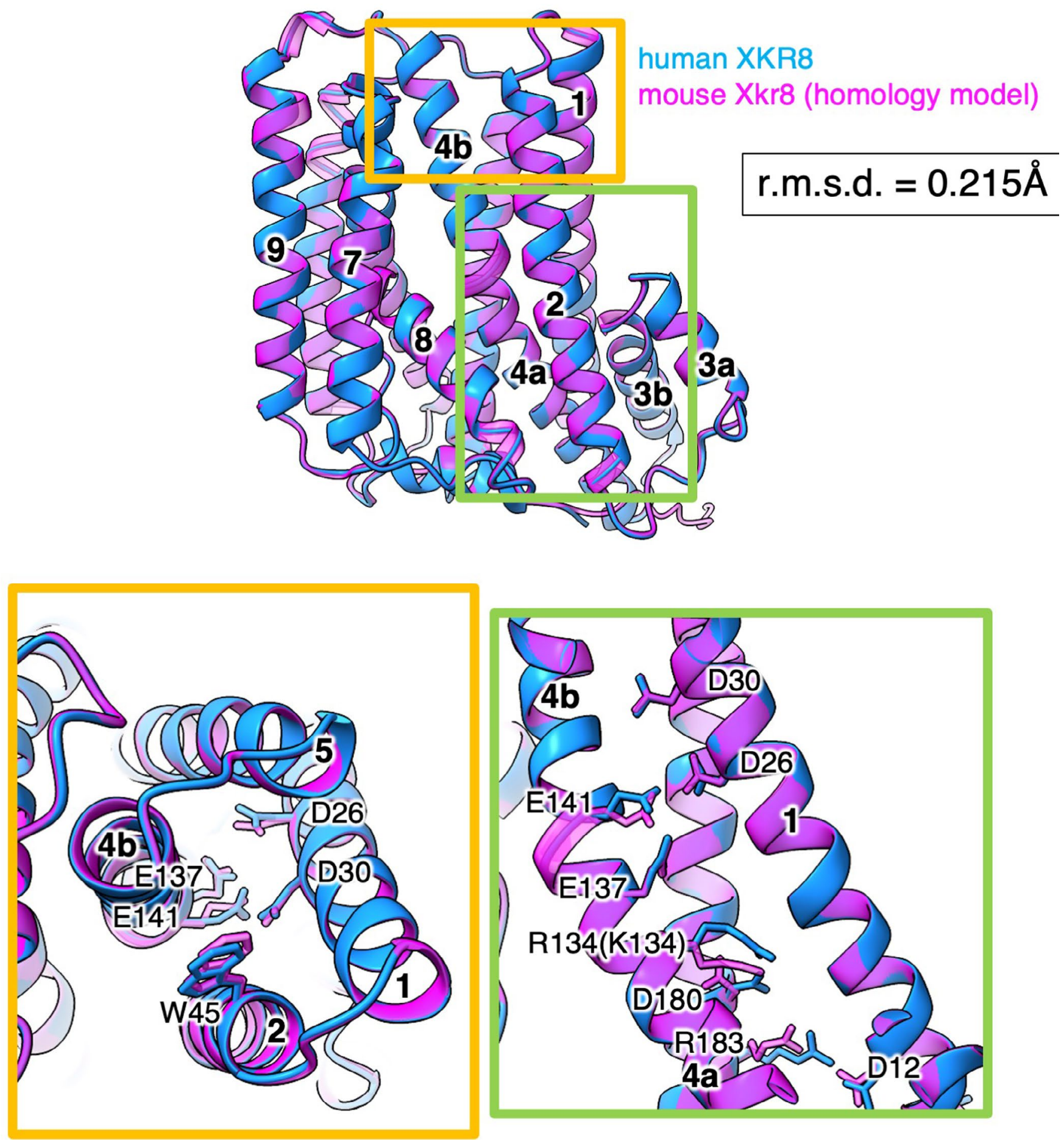

Extended Data Fig. 7 | Homology model of the $m X k r 8$ structure. The $m X k r 8$ homology model was predicted with MODELLER ${ }^{33}$ using the $h X k r 8$ structure (PDB:7DCE) as a template. It was superimposed on the $\mathrm{h} X \mathrm{kr} 8$ structure with a root mean square deviation (r.m.s.d.) of $0.215 \AA$. The area containing the residues involved in scrambling phospholipids at the extracellular side (orange box) and those in the middle of the membrane (green box) are enlarged in the lower panels. 


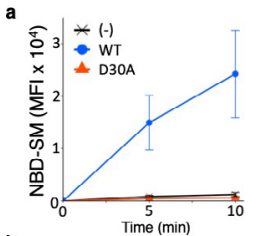

b

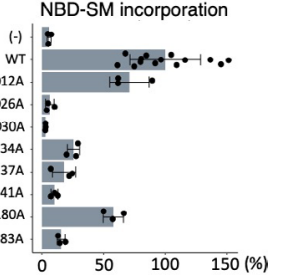

Extended Data Fig. 8 | Charged residues for the internalization of NBD-SM. Ba/F3 cells were transformed with wild-type or indicated $m$ Xkr8 mutants. Parental Ba/F3 and its transformants were incubated at $4{ }^{\circ} \mathrm{C}$ with NBD-SM for the indicated periods (a) or 5 min (b), treated with lipid-free BSA, and analyzed by flow cytometry. In $\mathbf{a}$, incorporated NBD-SM was analyzed by flow cytometry. Mean Fluorescence Intensity was regarded as inward scrambling activity. Experiments were performed three times. Data are presented as mean values + S.D. In $\mathbf{b}$, the scrambling activity of each mutant was normalized by its expression level estimated by GFP fluorescence. Experiments were performed three times. The data, relative activities to that of the wild-type, are presented as mean values \pm S.D. Source data for $\mathbf{a}$ and $\mathbf{b}$ are available online. 
a

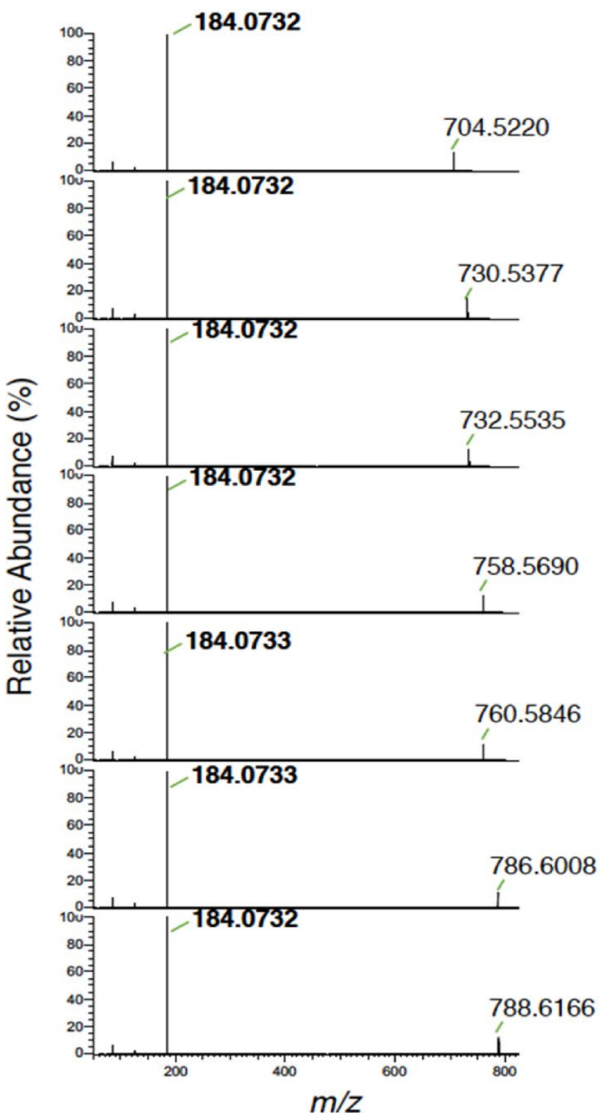

Positive

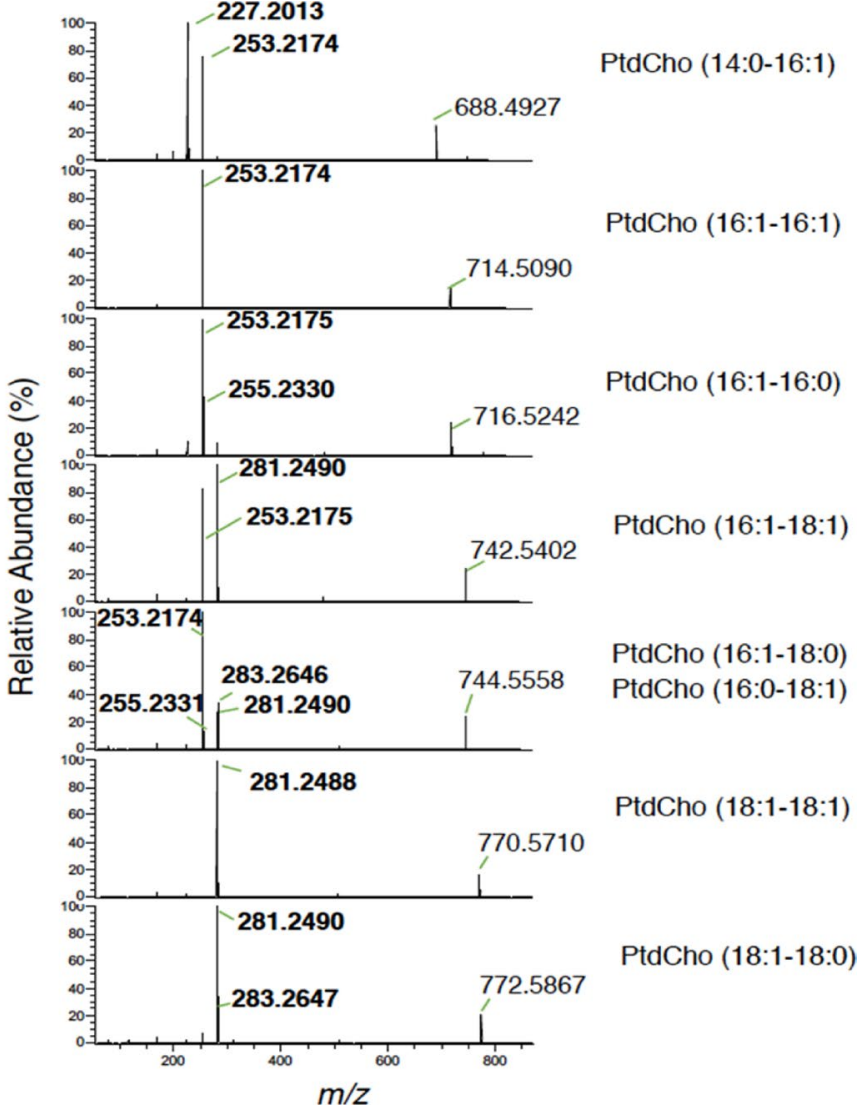

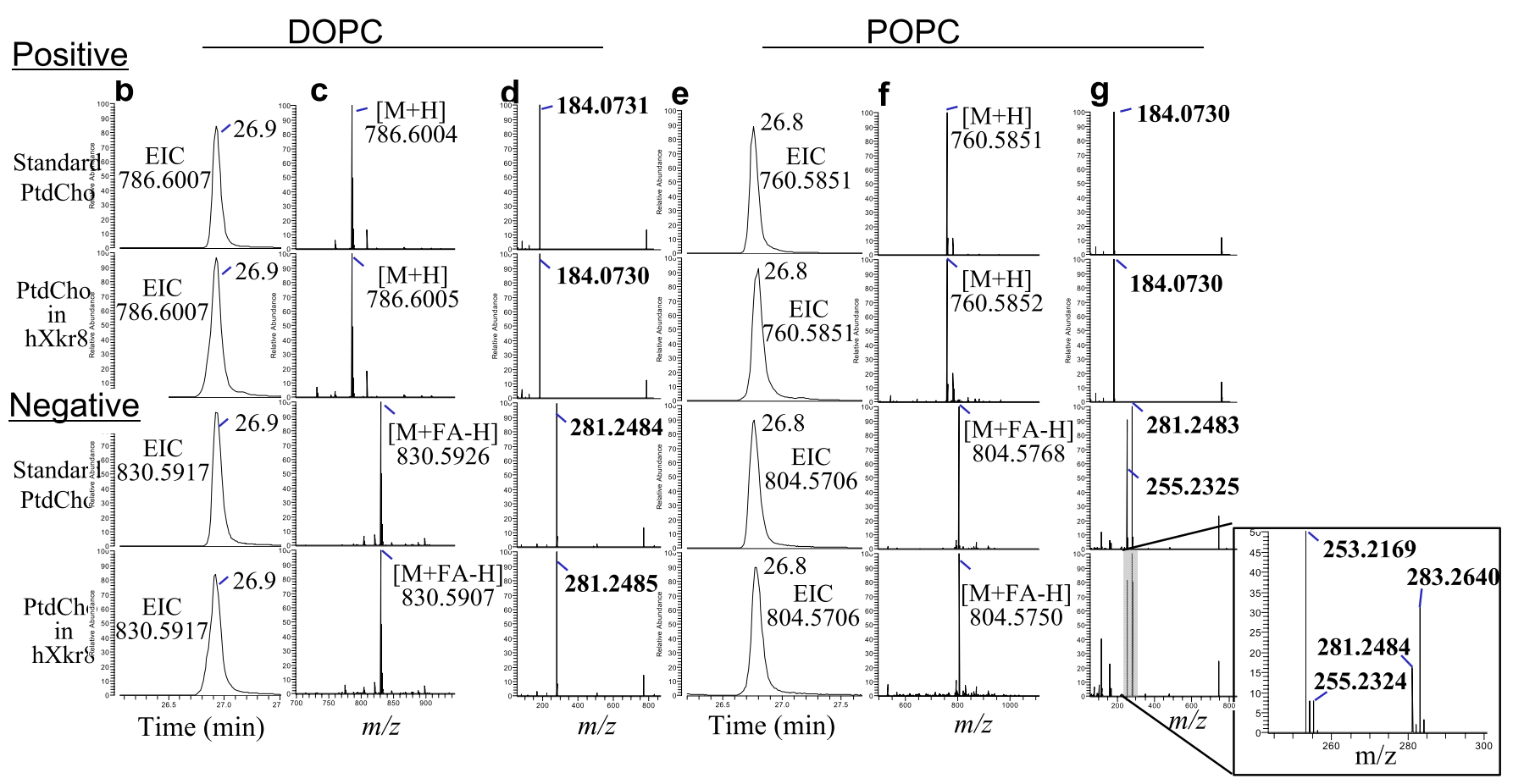

Extended Data Fig. 9 | See next page for caption. 
Extended Data Fig. 9 | MS/MS spectra of PtdCho species from the hXkr8-hBSG $\Delta$ complex. a, The MS/MS spectra of PtdCho species extracted from the hXkr8-hBSG $\Delta$ complex (hXkr8) in positive (left) and negative (right) ionization modes. The numbers in bold represent the $\mathrm{m} / \mathrm{z}$ values of fragment ions specific for each phospholipid and fatty acid class. In the positive ionization mode, a prominent peak at $m / z 184.073$ was assigned to phosphocholine ${ }^{68}$. In the negative ionization mode, the fatty acid composition of PtdCho was identified by the $m / z$ values of fragment ions. $\mathbf{b}-\mathbf{g}$, Data-dependent LC-MS/ MS was performed in positive and negative ionization modes with PtdCho standards, DOPC (18:1-18:1) (b-d) and POPC (16:0-18:1) (e-g), and PtdCho species from $\mathrm{hXkr8}$. b,e, Extracted ion chromatograms (EICs) of the indicated $\mathrm{m} / z$ values corresponding to theoretical $\mathrm{m} / \mathrm{z}$ of ionized DOPC and POPC. c,f, Precursor ion spectra at 26.9 and $26.8 \mathrm{~min}$, respectively. $\mathbf{d}, \mathbf{g}$, Product ion spectra of the indicated $\mathrm{m} / \mathrm{z}$ precursor ions. Numbers in bold represent $\mathrm{m} / \mathrm{z}$ values of fragment ions specific for each phospholipid class and fatty acid class. 

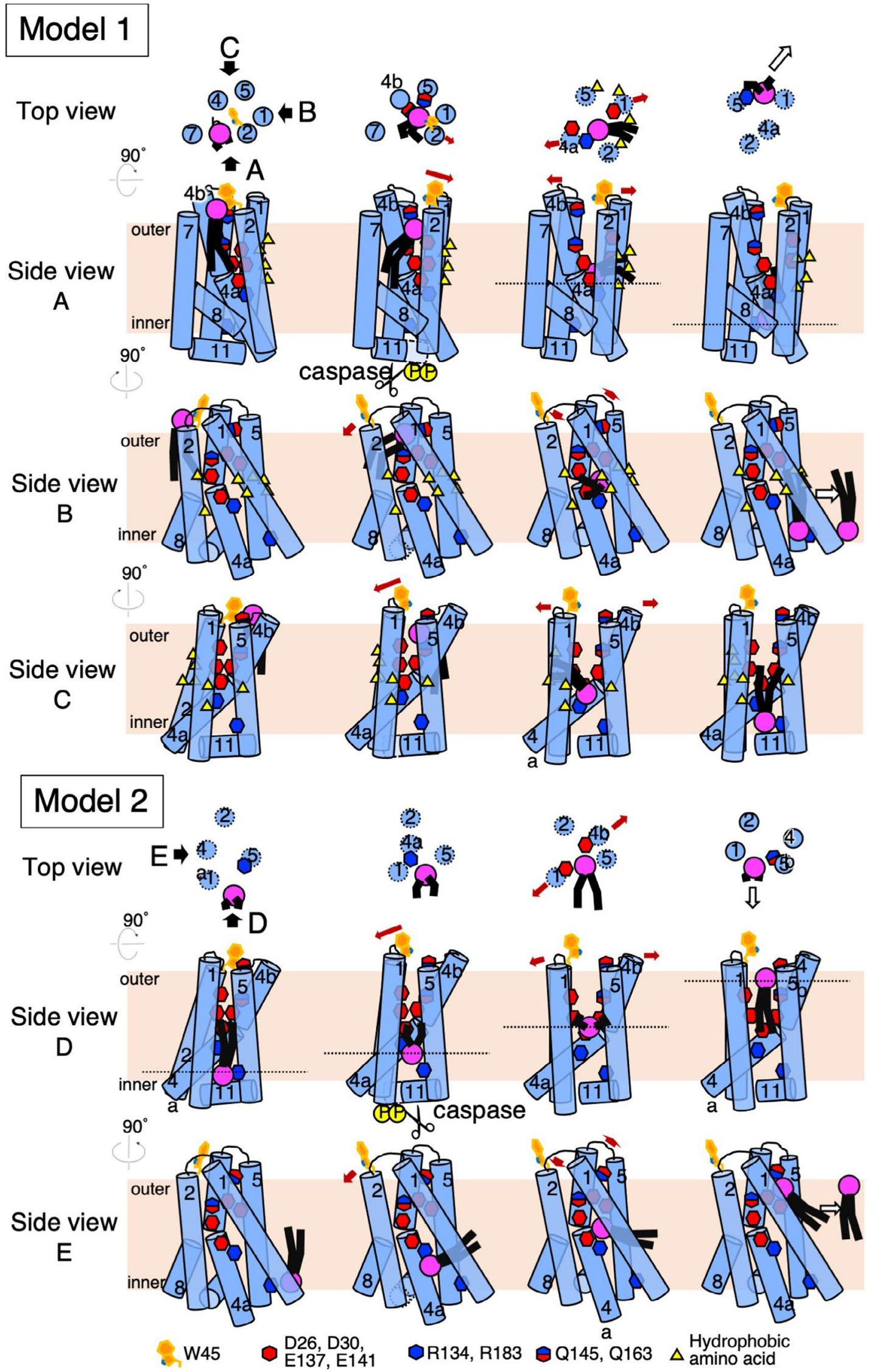

Extended Data Fig. 10 | See next page for caption. 
Extended Data Fig. 10 | Two models for Xkr8-mediated phospholipid scrambling. In the first model, a phospholipid enters the hydrophobic cleft between $\alpha 2$ and $\alpha 7$ (Step 1). Caspase 3 cleaves Xkr8 in the middle of $\alpha 11$ or a putative kinase(s) phosphorylates residues at the C-terminal tail region, which causes the exterior bending of $\alpha 2$ and removes W45 from the path. It causes the opening of the upper space between $\alpha 2$ and $\alpha 4 b$. The head group of the phospholipid enters the upper area of the track, and its interaction with Q163 and Y37 may open the hydrophilic path (Step 2). The contact of the phosphate group with acidic residues (D26, D30, E137, and E141) in the track generates the repulsion force and widens the pathway for the phospholipid to move. The hydrophobic residues (F23, L27, L31, V49, L50, and L53) between $\alpha 1$ and $\alpha 2$ facilitate the movement of acyl groups (Step 3). The phospholipid moves to the cytoplasmic site by interacting with positively charged residues (R134 and R183) and leaves the molecule between $\alpha 1$ and $\alpha 5$ (Step 4). In this model, the movement of phospholipids from the outer to inner leaflets opens the pathway. The second model is similar to the credit card model ${ }^{23}$. When $\mathrm{Xkr} 8$ is activated, the cytoplasmic space between $\alpha 1$ and $\alpha 5$ becomes wider, and the phospholipid head group is recruited into the space via positively charged residues (R134 and R183), leaving the acyl groups in the membrane area (Steps 1 and 2). The contact of the negatively charged head group with acidic residues (D26, D30, E137, and E141) broadens the upper space between $\alpha 1$ and $\alpha 5$ (Step 3), and the phospholipid leaves the molecule (Step 4). In this model, the hydrophobic cleft between $\alpha 2$ and $\alpha 7$ is not for phospholipid entry, and the PtdCho in the aperture stabilizes the molecule. 


\section{Reporting Summary}

Nature Research wishes to improve the reproducibility of the work that we publish. This form provides structure for consistency and transparency in reporting. For further information on Nature Research policies, see our Editorial Policies and the Editorial Policy Checklist.

\section{Statistics}

For all statistical analyses, confirm that the following items are present in the figure legend, table legend, main text, or Methods section.

$\mathrm{n} / \mathrm{a}$ Confirmed

$\bigotimes$ The exact sample size $(n)$ for each experimental group/condition, given as a discrete number and unit of measurement

Х A statement on whether measurements were taken from distinct samples or whether the same sample was measured repeatedly

X The statistical test(s) used AND whether they are one- or two-sided

Only common tests should be described solely by name; describe more complex techniques in the Methods section.

$\triangle \square$ A description of all covariates tested

Х $\square$ A description of any assumptions or corrections, such as tests of normality and adjustment for multiple comparisons

A full description of the statistical parameters including central tendency (e.g. means) or other basic estimates (e.g. regression coefficient) AND variation (e.g. standard deviation) or associated estimates of uncertainty (e.g. confidence intervals)

$\square$ For null hypothesis testing, the test statistic (e.g. $F, t, r$ ) with confidence intervals, effect sizes, degrees of freedom and $P$ value noted

Х Give $P$ values as exact values whenever suitable.

Х $\square$ For Bayesian analysis, information on the choice of priors and Markov chain Monte Carlo settings

Х $\square$ For hierarchical and complex designs, identification of the appropriate level for tests and full reporting of outcomes

Х $\square$ Estimates of effect sizes (e.g. Cohen's $d$, Pearson's $r$ ), indicating how they were calculated

Our web collection on statistics for biologists contains articles on many of the points above.

\section{Software and code}

Policy information about availability of computer code

Data collection SHIKA as of October 2018, KUMA as of October 2018

Data analysis autoPROC v1.0.5, STRANISO v2.3.36, CCP4 v7.1, Molrep v11.7.03, Refmac v5.8.0267, PHENIX v1.19rc2 4022, Coot v0.9, CTFFIND v4, RELION v3.1, RStudio v1.1.463, PyMol v2.4.0, UCSF Chimera v1.13.1, UCSF ChimeraX v0.93, FlowJo7.6.5, FACSDiva v6.1.3

For manuscripts utilizing custom algorithms or software that are central to the research but not yet described in published literature, software must be made available to editors and

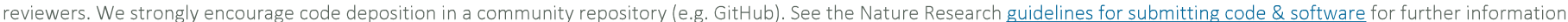

\section{Data}

Policy information about availability of data

All manuscripts must include a data availability statement. This statement should provide the following information, where applicable:

- Accession codes, unique identifiers, or web links for publicly available datasets

- A list of figures that have associated raw data

- A description of any restrictions on data availability

The cryo-EM density map for hXkr8-hBSG $\triangle$-Fab18 was deposited in the Electron Microscopy Data Bank (accession number: EMD-30636). The coordinates for the models of the hXkr8-hBSG $\triangle$-Fab18 complex were deposited in the Protein Data Bank (PDB) under the accession code PDB 7DCE. The coordinates and structural factors of Fab14 and the hBSG (domain2)-Fab14 complex were deposited in PDB under accession codes PDB 7D9Z and PDB 7DAA, respectively. The mass spectrometry data was deposited in the ProteomeXchange Consortium under accession code PXD027776. All other data are available from the corresponding authors upon reasonable request. Several structural coordinates in the PDB database were used in this study, which can be located by accession numbers $3 \mathrm{~B} 5 \mathrm{H}$, $4 \mathrm{ma} 3$, and $1 \ln 2$. 
Please select the one below that is the best fit for your research. If you are not sure, read the appropriate sections before making your selection.

$\bigotimes$ Life sciences $\quad \square$ Behavioural \& social sciences $\quad \square$ Ecological, evolutionary \& environmental sciences

For a reference copy of the document with all sections, see nature.com/documents/nr-reporting-summary-flat.pdf

\section{Life sciences study design}

All studies must disclose on these points even when the disclosure is negative.

Sample size Sample sizes were not pre-determined. X-ray diffraction and Cryo-EM images were collected until structures of satisfactory quality were solved, which suggested sufficient sample size. For biochemical and cellular experiments, no information was derived about a population based on sampling, and therefore sample size determination was not necessary.

Data exclusions No data were excluded.

Replication For all experiments presented as representative images in Fig. 1a and 1b, Fig. 2e, 3b, $5 \mathrm{~g}$ and Extended Data Fig. 1b, 3-5 biological replicates were performed. The experiments presented in Fig. $2 \mathrm{c}$ and Fig. $3 \mathrm{c}$ were performed twice. The experiments presented in Fig. 1a (SDS-PAGE) and Extended Data Fig. 1d and 1e were performed more than 5 times. All other experiments were performed at least three times and described in legend for each Figure.

Randomization No group allocation was performed in this study

Blinding Blinding was not performed as subjective analysis was not needed. Each experiment was analyzed using consistent methods. Quantitative measurements using various approaches and reaction kits as described in the methods minimized biased assessments.

\section{Reporting for specific materials, systems and methods}

We require information from authors about some types of materials, experimental systems and methods used in many studies. Here, indicate whether each material, system or method listed is relevant to your study. If you are not sure if a list item applies to your research, read the appropriate section before selecting a response.

\begin{tabular}{l|l} 
Materials \& experimental syste \\
\hline$n / a$ & Involved in the study \\
$\square$ & $\bigotimes$ Antibodies \\
$\square$ & $\square$ Eukaryotic cell lines \\
$\square$ & $\square$ Animals and other organisms \\
$\square$ & $\square$ Clinical data \\
$\square$ & $\square$ Dual use research of concern
\end{tabular}

\begin{tabular}{l|l}
\multicolumn{2}{l}{ Methods } \\
\hline n/a & Involved in the study \\
$\square$ & $\square$ ChIP-seq \\
$\square$ & $\bigotimes$ Flow cytometry \\
$\square$ & $\square$ MRI-based neuroimaging
\end{tabular}

\section{Antibodies}

Antibodies used

Rabbit HRP-anti-GFP antibody (Anti-GFP pAb-HRP-DirecT) MBL Cat\# 598-7 (1: 10000)

Mouse HRP-anti-HA mAb (Direct-Blot HRP anti-HA.11 Epitope Tag antibody) (Clone 16B12) BioLegend Cat\#901519 (1:3000)

Rabbit mAb against human Xkr8-Basigin complex (established in this report) (Clones XBA14 and XBA18)

Validation

HRP-labeled rabbit anti-GFP Ab was validated by MBL for Western blot (https://ruo.mbl.co.jp/bio/e/dt//A/?pcd=598-7) and previous publications (PMID:23840565, 26272249, 27578797, 27707755, 28369861, 28384198, 28588310, 30367048, and 32866183). HRPlabeled mouse anti-HA mAb was validated by BioLegend for Western blot (https://www.biolegend.com/en-us/products/direct-blothrp-anti-ha11-epitope-tag-antibody-14398?GroupID=GROUP26) and previous publications (PMID: 28827538, 28598330, 28082682, 28087701, 28053031, 28069948, 27909245, 27797818, 27834731)

Rabbit anti-human Xkr8-BSG mAb (clones XBA14 and XBA18), established in this study, was validated by Flow cytometry with mouse cell transformants expressing or not-expressing human Xkr8-Bsg complex. The Cryo-EM image of Xkr8-Bsg-Fab (XBA18) confirmed the binding of this mAb to the Xkr8-Bsg complex.

\section{Eukaryotic cell lines}

Policy information about cell lines

Cell line source(s)

HEK293T (ATCC CRL-3216) 
Authentication

Mycoplasma contamination

Commonly misidentified lines (See ICLAC register)

\section{None of the cell lines used were authenticated}

All cell lines were negative for mycoplasma.

Not used

\section{Animals and other organisms}

Policy information about studies involving animals; ARRIVE guidelines recommended for reporting animal research

Laboratory animals

Wild animals

Field-collected samples

Ethics oversight

\section{New Zealand White (NZW) rabbits, male, age of 13 weeks}

This study did not involve wild animals.

This study did not involve samples collected from the field.

All animal studies were approved by the Animal Care and Use Committee of the Research Institute of Microbial Diseases, Osaka University, and Chugai Pharmaceutical Co.

Note that full information on the approval of the study protocol must also be provided in the manuscript.

\section{Flow Cytometry}

Plots

Confirm that:

X The axis labels state the marker and fluorochrome used (e.g. CD4-FITC).

\The axis scales are clearly visible. Include numbers along axes only for bottom left plot of group (a 'group' is an analysis of identical markers).

\All plots are contour plots with outliers or pseudocolor plots.

$\bigotimes$ A numerical value for number of cells or percentage (with statistics) is provided.

\section{Methodology}

Sample preparation

Instrument

Software

Cell population abundance

Gating strategy
DKO cells, Ba/F3 cells, and their derivatives were cultured in RPMI1640 containing $10 \%$ FCS, collected by centrifugation, and suspended in Annexin V-staining buffer.

\section{BD FACSCanto II (BD Biosciences)}

Data was acquired using BD FACSDiva software v6.1.3, and analysed using FlowJo7.6.5

In some cases, stable transformant expressing the GFP-tagged XKR8 were sorted for GFP.

Dead cells were omitted from the analysis by standard SSC/FFC gating, PI or SytoxRed

$\bigotimes$ Tick this box to confirm that a figure exemplifying the gating strategy is provided in the Supplementary Information. 